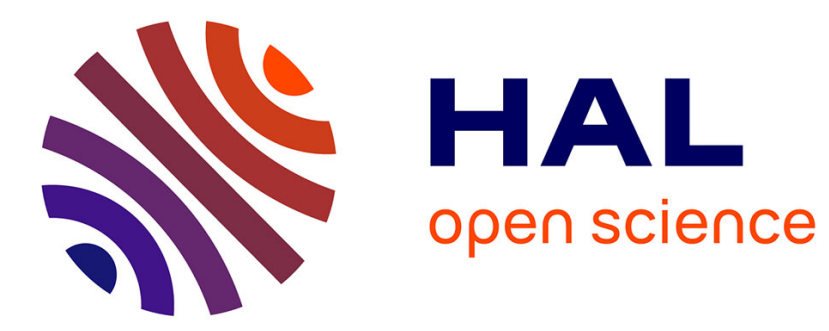

\title{
On the validity of the quasi-steady-turbulence hypothesis in representing the effects of large scales on small scales in boundary layers
}

Lionel Agostini, Michael Leschziner

\section{- To cite this version:}

Lionel Agostini, Michael Leschziner. On the validity of the quasi-steady-turbulence hypothesis in representing the effects of large scales on small scales in boundary layers. Physics of Fluids, 2016, 28 (4), pp.045102. 10.1063/1.4944735 . hal-03279534

\section{HAL Id: hal-03279534 \\ https://hal.science/hal-03279534}

Submitted on 6 Jul 2021

HAL is a multi-disciplinary open access archive for the deposit and dissemination of scientific research documents, whether they are published or not. The documents may come from teaching and research institutions in France or abroad, or from public or private research centers.
L'archive ouverte pluridisciplinaire HAL, est destinée au dépôt et à la diffusion de documents scientifiques de niveau recherche, publiés ou non, émanant des établissements d'enseignement et de recherche français ou étrangers, des laboratoires publics ou privés. 
See discussions, stats, and author profiles for this publication at: https://www.researchgate.net/publication/291354546

\section{Skewness-induced asymmetric modulation of small-scale turbulence by large-scale structures}

Article in Physics of Fluids · January 2016

DOI: 10.1063/1.4939718

3 authors, including:

Lionel Agostini

French National Centre for Scientific Research

50 PUBLICATIONS 512 CITATIONS

SEE PROFILE

Some of the authors of this publication are also working on these related projects:

Project $\quad$ Analysis of Deep Dynamic Stall View project 


\section{AIP | Physics of}

\section{Skewness-induced asymmetric modulation of small-scale turbulence by large-scale structures}

Lionel Agostini, Michael Leschziner, and Datta Gaitonde

Citation: Physics of Fluids 28, 015110 (2016); doi: 10.1063/1.4939718

View online: http://dx.doi.org/10.1063/1.4939718

View Table of Contents: http://scitation.aip.org/content/aip/journal/pof2/28/1?ver=pdfcov

Published by the AIP Publishing

\section{Articles you may be interested in}

Universality and scaling phenomenology of small-scale turbulence in wall-bounded flows

Phys. Fluids 26, 035107 (2014); 10.1063/1.4868364

High-Schmidt-number mass transport mechanisms from a turbulent flow to absorbing sediments Phys. Fluids 24, 085103 (2012); 10.1063/1.4739064

Anisotropy in pair dispersion of inertial particles in turbulent channel flow

Phys. Fluids 24, 073305 (2012); 10.1063/1.4737655

Influence of an anisotropic slip-length boundary condition on turbulent channel flow

Phys. Fluids 24, 055111 (2012); 10.1063/1.4719780

On the large-scale structure and spectral dynamics of two-dimensional turbulence in a periodic channel

Phys. Fluids 20, 056602 (2008); 10.1063/1.2919132

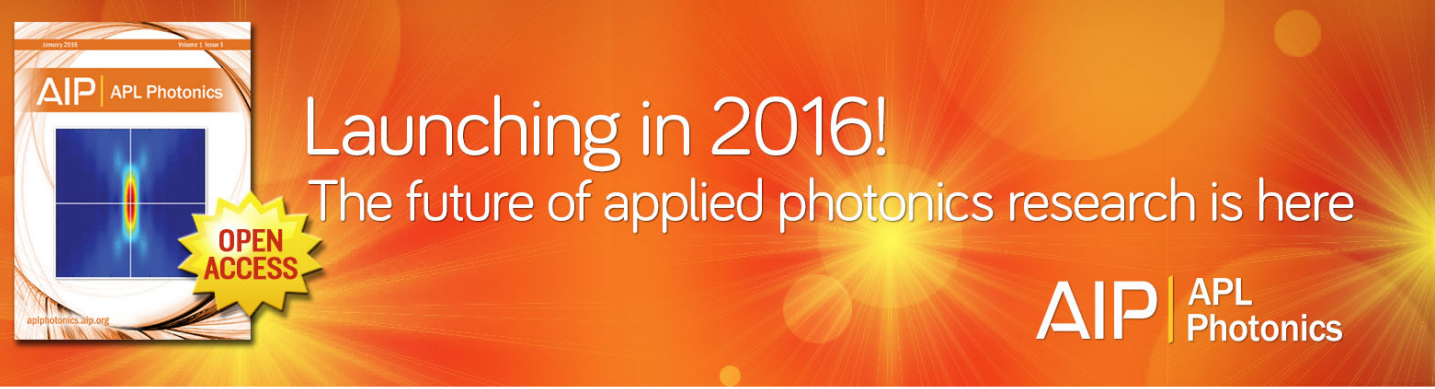




\title{
Skewness-induced asymmetric modulation of small-scale turbulence by large-scale structures
}

\author{
Lionel Agostini, ${ }^{1}$ Michael Leschziner, ${ }^{2}$ and Datta Gaitonde ${ }^{1}$ \\ ${ }^{1}$ Mechanical and Aerospace Engineering, The Ohio State University, Columbus, \\ Ohio 43210, USA \\ ${ }^{2}$ Department of Aeronautics, Imperial College London, South Kensington, \\ London SW7 2AZ, United Kingdom
}

(Received 1 October 2015; accepted 28 December 2015; published online 22 January 2016)

\begin{abstract}
Several recent studies discuss of role of skewness of the turbulent velocity fluctuations in near-wall shear layers, in the context of quantifying the correlation between large-scale motions and amplitude variations of small-scale fluctuations-referred to as "modulation." The present study is based on the premise that the skewness of the small-scale fluctuations should be accounted for explicitly in the process of defining their envelope, which characterizes their amplitude variations. This leads to the notion of two envelopes, one for positive and the other for negative small-scale fluctuations, and hence also to two corresponding correlation coefficients. Justification for this concept is provided first by an examination of a high-frequency synthetic signal subjected to realistic skewness-inducing modulation. A new formalism is provided for deriving the two envelopes, and its fidelity is demonstrated for the synthetic test case. The method is then applied to a channel flow at a friction Reynolds number of 4200, for which direct numerical simulation (DNS) data are available. The large-scale and small-scale fields are separated by the empirical mode decomposition method, and the modulation of the small-scale fluctuations by the large scales is examined. Separate maps of the correlation coefficient and of two-point correlations, the latter linking the large-scale motions and the envelopes of the small-scale motions, are derived for the two envelopes pertaining to positive and negative small-scale fluctuations, and these demonstrate a significant sensitivity to the envelope-definition process, especially close to the wall where the skewness of the small-scale fluctuations is the dominant contributor to the total value. (C) 2016 AIP Publishing LLC. [http://dx.doi.org/10.1063/1.4939718]
\end{abstract}

\section{INTRODUCTION}

A key aspect of the interaction between coherent large-scale and small-scale structures in a turbulent boundary layer is the degree to which the intensity of the former causes an amplification or attenuation of the intensity of the latter-a process referred to as "modulation." To examine the correlation between the large-scale motions and the modulation, the large-scale and small-scale fields need first to be separated. This may be achieved in various ways - typically, by some Fourier-based filtering with prescribed wavelength cutoff or, more rarely, and as the present authors elected to do, by the use of the Empirical Mode Decomposition (EMD) of Huang et al. ${ }^{1}$ (see the work of Agostini and Leschziner ${ }^{2}$ ).

In order to identify the large-scale-induced variation of the amplitude of the small-scale motions, the envelope of the fluctuations needs to be determined. This subject was first addressed by Bandyopadhyay and Hussain ${ }^{3}$ and more recently elaborated on by Mathis et al., ${ }^{4}$ who have advocated the use of the Hilbert transform to define the envelope, either for the streamwise component only ${ }^{4,5}$ or all three components. ${ }^{6}$ The currently accepted procedure involves two major steps:

1. With $u_{s s}$ being the small-scale-fluctuation field and $\breve{u}_{s s}$ denoting its Hilbert transform, an augmented signal $z=u_{s s}+i \breve{u}_{s s}$ is defined, and the modulus of this signal $|z|$ is estimated in order to determine envelope of the small-scale signal. 
2. The envelope so determined is intended to reflect only the effects of the largest modulating scales. However, in reality, the actual outcome of the transformation is a signal that is 'contaminated' by higher-frequency components that arise from a broad range of scales that lie between those associated with the small-scale motions and the large-scale motions whose modulating effects are to be captured. This necessitates a low-pass filtering of the envelope, so as to eliminate the intermediate scales, yielding the signal $|z|_{L F}$.

The second step reflects the assumption that the fluctuations eliminated by the filtering are entirely unrelated to the large-scale motions. The target is thus a smooth signal that is held to uniquely represent the variations of the amplitude-modulated small-scale signal. With this envelope so determined, its correlation with the large-scale component can be addressed.

The present contribution is intended to provide justification for the claim that the above, conventional, two-stage approach to defining the envelope fails to take into account the skewness of the modulated small-scale signal, which is a potentially influential physical phenomenon. Similarly, an alternative method proposed by Chung and McKeon ${ }^{7}$ and Jacobi and McKeon, ${ }^{8}$ in which the small-scale energy is recorded using a moving-average filter, does not provide any information about how the large scales alter the positive and negative parts of the small-scale signals, separately, due to skewness. The above envelope-definition methods thus require a fundamental correction, which is provided herein. It is argued, in particular, that the insensitivity of the definition process to the skewness prejudices the evaluation of the correlation between the large-scale motion and the envelope.

The importance of skewness in the present context was previously discussed and analysed by Schlatter and Örlü, ${ }^{9}$ Bernardini and Pirozzoli, ${ }^{10}$ Mathis et al. ${ }^{11}$ and Duvvuri and McKeon, ${ }^{12}$ in light of the observed similarity between the wall-normal variations of correlation coefficient and the skewness of the total streamwise fluctuations. Schlatter and Örlü ${ }^{9}$ argue that the envelope-definition approach advocated by Mathis et al. ${ }^{4,13}$ does not represent a robust tool for determining the correlation in the presence of skewness. Based on a numerical-modulation experiment with a synthetic signal, they claim to quantify the relationship between the correlation coefficient and the skewness. However, this explanation may be contested on the grounds that it is based on a modulating signal in which the large-scale fluctuations are purely a consequence of skewness variations-i.e., the skewness does not vary relative to the low-pass-filtered signal that is presumed to represent the large-scale motion. The inevitable consequence is then a physically unrealistic dependence of the correlation coefficient on the skewness.

In the light of the observations of Schlatter and Örlü, Bernardini and Pirozzoli proposed to characterize the modulation by way of the two-point correlation between the envelope of the modulated near-wall small-scale field and the energetic large-scale motions in the outer layer, arguing that this approach provides a superior representation of the effects of the outer structures on the near-wall modulation of the small-scale fluctuations, also claimed to bypass the need to account for the dependence of the correlation on the skewness. However, as will be shown in Section V, this is not borne out by the present study, due to the dominance of the small-scale skewness among the contributions to the total level of the skewness.

Mathis et al. ${ }^{11}$ focused, principally, on the four triple-correlation fragments of large-scale and small-scale fluctuations contributing to the total skewness, extending Schlatter and Örlü's consideration of the mixed-correlation terms only. Predictably, the only mixed term that is of any significance is the one that correlates large-scale fluctuations with the square of the small-scale fluctuations. In fact, this term can be interpreted as being an approximate representative of the correlation between large-scale motions and the envelope of the modulated small-scale signal. The only other important fragment is the skewness of the small-scale fluctuations. In fact, this fragment is the strongly dominant contributor to the total skewness near the wall, as will be illustrated by results to follow. In contrast, in the outer region, where scale-separation is not well defined, and is also quite sensitive to the large-scale/small-scale separation (filtering) process, the correlations involving the large-scale motions make substantial contributions to the full-field skewness. These observations support the present contention that the skewness of the small-scale motions is an important property to consider when quantifying the near-wall modulation process. In the outer region, this focus may 
not be productive, but in that region, the distinction between small-scale and large-large motions is tenuous in any event.

In a recent study, Duvvuri and McKeon ${ }^{12}$ examine the relationship between the skewness of the total-fluctuation field and the correlation coefficient by way of exact manipulations of a general Fourier representation of the streamwise-velocity signal-an approach that does not account for dynamic interactions, however. They show that only certain triadic combinations of the wave number contribute to the skewness of the total signal, and they proceed to deriving a relationship that links the correlation coefficient to the skewness, the former utilizing the standard envelope-definition methodology of Mathis et al. ${ }^{4}$ Duvvuri and McKeon ${ }^{12}$ thus argue that their analysis, supported by a specifically targeted experiment, explains the correspondence between the correlation coefficient and the skewness. However, this study does not address the link between the correlation coefficient and the dominant small-scale skewness, nor the effects of the latter on the envelope definition.

In what follows, the validity of the Hilbert-transform approach, as a basis for quantifying the modulated small-scale signal, is re-examined be reference to synthetic signals and DNS data for a channel flow at $R e_{\tau}=4200 .{ }^{14,15}$ The analysis of the synthetic signals shows that the envelope of the small-scale motions, determined with the Hilbert-transform, can be very sensitive to the skewness of the former. In real near-wall turbulence, the skewness is dependent, in turn, on the sign of the large-scale fluctuations and thus on whether these fluctuations are associated with sweeps or ejections. The conclusion emerging from the analysis is that the definition of an envelope, by use of low-pass filtering, leads to an important loss of information that is closely associated with the effects of the skewness of the small-scale field on the modulation process. An improved Hilbert-transform-based method is then proposed to characterize the modulation. This method is then contrasted with the conventional approach when both are applied to a channel flow at $R e_{\tau}=4200$ for which DNS data have been provided by Lozano-Durán and Jiménez. ${ }^{14,15}$

\section{THE LINK BETWEEN SKEWNESS OF THE SMALL-SCALE FLUCTUATIONS AND SPLATTING}

Agostini and Leschziner ${ }^{2}$ have highlighted the fact that the small-scale (SS) modulation by the large-scale (LS) motions is not symmetric. In particular, they provided evidence for the fact that, unlike the linear modulation of the negative SS fluctuations, the positive fluctuations are altered by a "splatting" effect, which manifests itself by a decrease in the positive streamwise fluctuations and an increase in the spanwise fluctuations-in statistical terms, representing an energy-redistribution process by pressure-velocity interaction. This is exemplified in Figure 1(b) by conditionally sampled joint $\left(u_{S S}^{+}-w_{S S}^{+}\right)$probability-density functions (PDFs) for the SS motions in the channel flow at $R e_{\tau}=4200$ at $y^{+} \approx 12.5$, one (red contours) obtained in regions characterized by the largest $10 \%$ of positive LS fluctuations, and the other (blue contours) applicable to the lowest $10 \%$ of negative LS fluctuations, identified, respectively, by the right-hand-side and left-hand-side tails of the LS PDF. The joint PDFs illustrate two facts: First, the pear-shaped contours reflect the process of SS splatting, associated with sweeps and positive SS fluctuations; second, the intensity of the SS splatting depends on the sign of the LS fluctuations-positive fluctuations, associated with LS sweeps, causing more severe distortions of the SS PDF.

The single-component PDFs for $u_{S S}^{+}$and $w_{S S}^{+}$, given in Figures 1(c) and 1(d), respectively, accentuate the sensitivity of the SS motions to the sign of the LS fluctuations. The chain (blue) lines relate to the blue PDF contours in Figures 1(a) and 1(b), i.e., to the joint PDFs associated with negative LS motions, while the solid (red) lines relate to the red contours, i.e., to joint PDFs associated with positive LS motions. The PDFs are normalized in such a way that their integral is equal to 100 . The most important feature to highlight is the substantial difference in the level of skewness of the PDFs for $u_{S S}^{+}$, which reflects the sensitivity to the sign of the LS motions. Negative LS fluctuations, associated with ejections, not only lead to narrower PDFs, but also lead to much lower levels of skewness of the SS fluctuations.

Wall-normal variations of the skewness and flatness (Kortosis) of the conditional PDFs of $u_{S S}^{+}$ and $w_{S S}^{+}$are given in Figures 2(a) and 2(b), respectively. Each figure contains three curves: the blue relating, again, to extreme negative LS fluctuations, and the red to extreme positive LS fluctuations. 


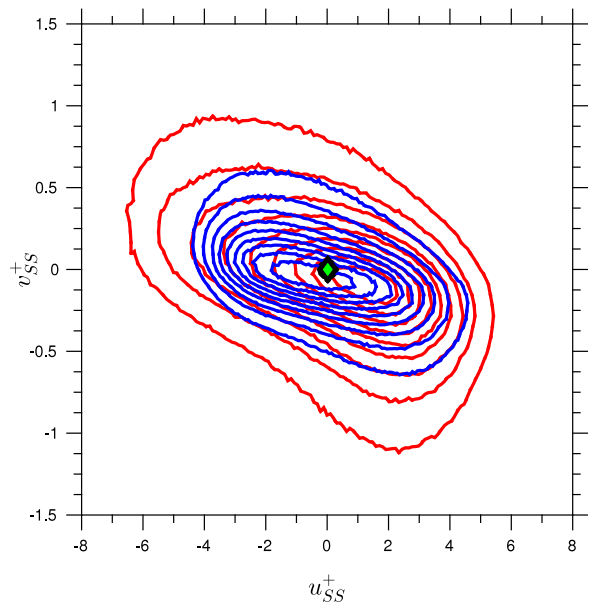

(a)

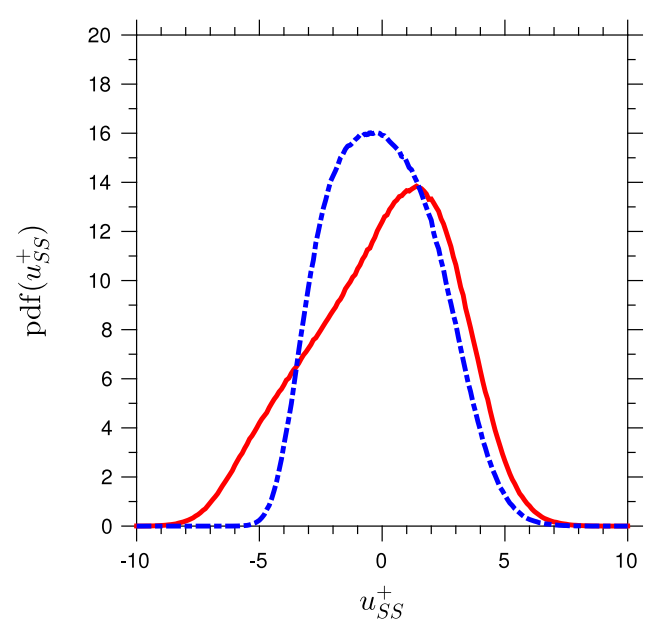

(c)

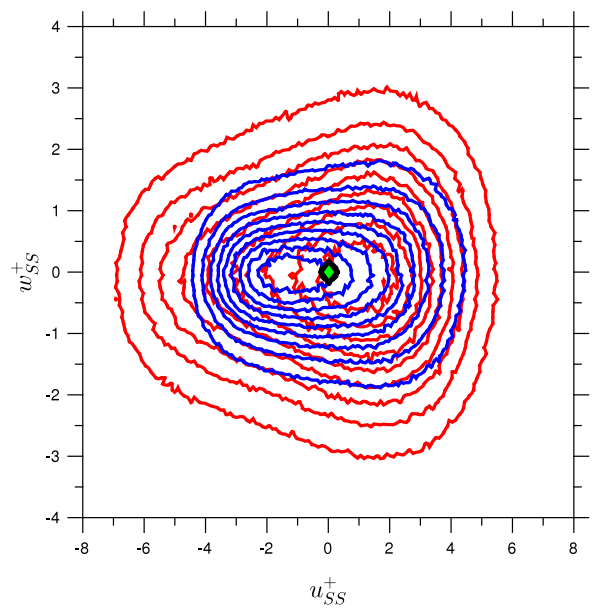

(b)

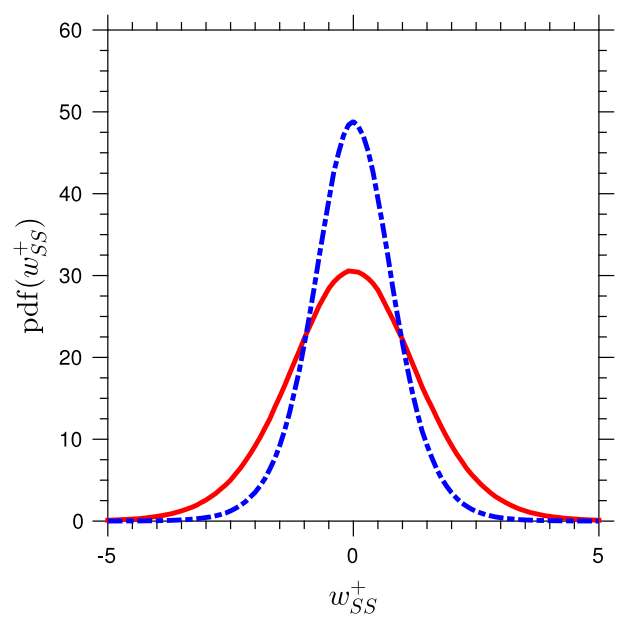

(d)

FIG. 1. PDFs of small-scale fluctuations, normalized by the mean friction velocity, conditional on regions of $10 \%$ extreme positive and negative large-scale fluctuations (red and blue lines, respectively) in channel flow at $R e_{\tau}=4200$ and $y^{+} \approx 12.5$. (a) $\left(u_{S S}^{+}-v_{S S}^{+}\right)$PDFs; (b) $\left(u_{S S}^{+}-w_{S S}^{+}\right)$PDFs; (c) $u_{S S}^{+}$PDFs; (d) $w_{S S}^{+}$PDFs.

The black curves relate to regions defined by the lowest magnitude of LS motions, within the central $10 \%$ area of the PDF of the LS fluctuations. Above $y^{+} \approx 10$, the skewness of the SS PDFs associated with positive LS fluctuations is much larger than that for negative LS fluctuations. In this region, the PDFs of $w_{S S}^{+}$are close to being Gaussian, indicated by a Kurtosis level close to 3 . Below $y^{+} \approx 10$, the behaviour changes drastically: the skewness reverses in sign, is significantly less sensitive to the sign of the LS motions, and is associated with high Kurtosis values of the PDF of $w_{S S}^{+}$, the last feature indicating a narrow, peaky distribution with heavy tails of high-intensity spanwise fluctuations.

The reversal in the skewness across $y^{+} \approx 10$ is associated with the change in sign, across the buffer region, in the wall-normal convective transport of SS fluctuations by dominant sweeping events of LS motions. With the maximum SS intensity highest in the buffer layer, these strong sweep events, relative to more numerous but weaker ejection events, tend to carry low-SS-intensity fluid towards to wall above the buffer layer and high-SS-intensity fluid towards the wall below the buffer layer. In statistical terms, turbulent transport, represented by triple corrections of the form $\overline{v u u}$, shifts streamwise energy from the peak-energy (and production) buffer region towards, as well as away from, the wall, which again implies a change in sign across the buffer layer. While the distortions in the PDFs beyond $y^{+} \approx 10$ is dominated by the effects of the LS motions, the steep rise 


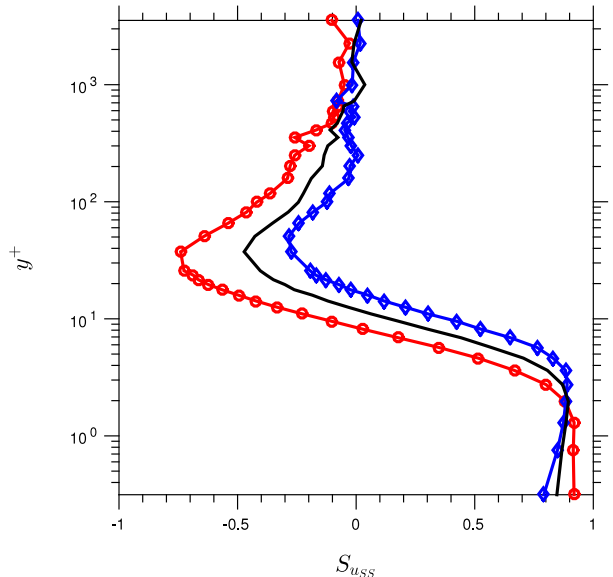

(a)

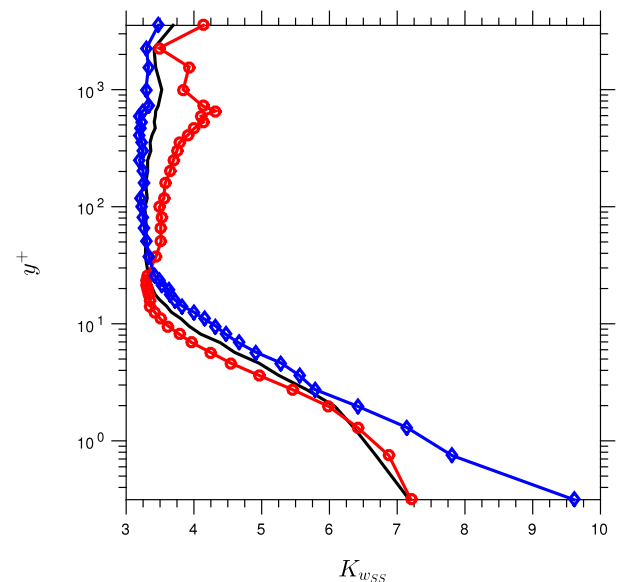

(b)

FIG. 2. Wall-normal variations of the skewness and flatness of conditional PDFs for channel flow at $R e_{\tau}=4200$. (a) Skewness of $u_{S S}^{+}$PDFs and (b) flatness of $w_{S S}^{+}$PDFs. Blue and red lines relate, respectively, to extreme $10 \%$ positive and $10 \%$ negative LS fluctuations; black lines arise from conditioning to the $10 \%$ weakest LS fluctuations.

in the flatness of the $w_{S S}^{+}$fluctuations and the declining sensitivity of SS fluctuations to LS motions as the wall is approached are indicative of a strengthening direct interaction between the small-scale motions and the wall. Splatting by SS motions intensifies, and convective transport of streamwise SS fluctuations by wall-normal SS sweeps causes a heavy positive tail in the PDF of $u_{S S}^{+}$close to the wall.

The most important message to carry forward is that the skewness of the SS motions is an important property. Indeed, it is a key distinguisher between the SS statistics arising for positive and negative LS fluctuations, associated with sweeps and ejections, respectively.

\section{DETERMINATION OF THE SS ENVELOPE}

\section{A. Small-scale modulation}

The nature of modulation is illustrated here by reference to a snapshot of the spanwise distribution of the streamwise velocity taken from the channel flow DNS at $R e_{\tau}=4200$ and $y^{+} \approx 12.5$. Figure 3(a) shows the total signal, in black, and the LS component, in red, as determined from a bi-dimensional version of the EMD adapted from the generic framework proposed by Huang et al. ${ }^{1}$ The effectiveness of the EMD, as an approach to the decomposition of the fluctuations, is demonstrated by Agostini and Leschziner ${ }^{2}$ and Agostini et al. ${ }^{16}$ Figure 3(b) shows the SS component-the difference between the two signals in Figure 3(a) — and the LS signal, the solid red line, artificially shifted upwards by $\left[\min \left(u_{L S}^{+}\right)+1\right]$. The LS signal is included twice, the lower dashed red line being a mirror image of the upper solid line. Visual inspection of the SS fluctuations reveals, qualitatively, a distinct correlation between the LS fluctuations and amplitude variations of the SS signal on the negative side. This correlation is referred to as "modulation," as described by Marusic and his co-workers. ${ }^{4,5}$ In contrast, for positive SS fluctuations, the correlation appears to be far weaker. The fact that there are significant differences is not surprising, in view of the foregoing discussion on the importance of the skewness, brought to light by the conditional PDFs. The implication is, therefore, that a single envelope of the SS motions may not be adequate, and that envelopes need to be defined separately for negative and positive SS fluctuations, with the skewness taken into account.

\section{B. The role of skewness-a toy model}

In order to justify the assertion that the derivation of the SS envelope by Mathis et al. ${ }^{4}$ requires revision, and to illustrate the rationale of the alternative approach proposed herein, a synthetic signal 


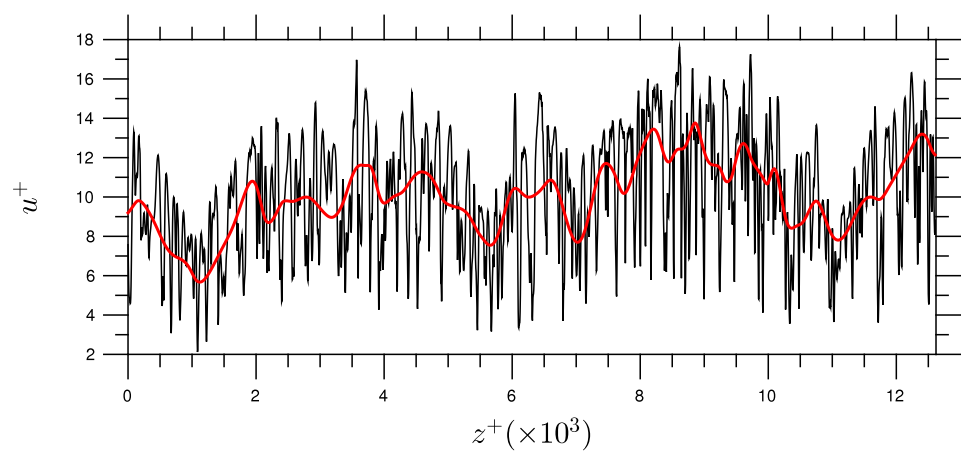

(a)

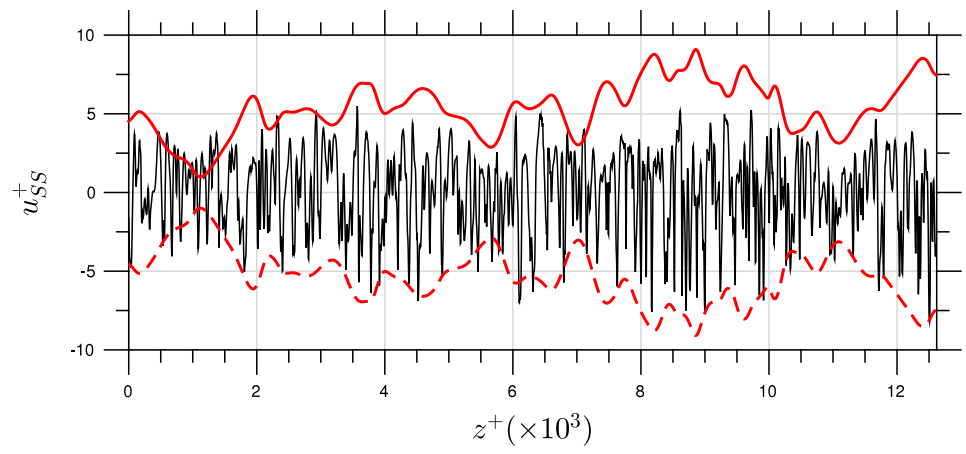

(b)

FIG. 3. Snapshot of the turbulent streamwise motion along a spanwise line taken from the channel-flow DNS at $R e_{\tau}=4200$ and $y^{+} \approx 12.5$. (a) Full signal (black line) and large-scale signal (red line); (b) small-scale fluctuations (black line), large-scale fluctuations, shifted upwards by $\left[\min \left(u_{L S}^{+}\right)+1\right]$ (solid red line), and its mirror image (dashed red line).

is considered below, wherein the SS signal is represented by a pure high-frequency sinusoidal function $x\left(z_{s}\right)$ being modulated by the signal $e\left(z_{s}\right)=f\left(u_{L S}\right)$, with $u_{L S}$ taken from the actual channel-flow data-the red line in Figure 3-subject to $z_{s}=z^{+}$. In plots to follow in this section for synthetic signals, the generic coordinates $\phi$ and $z_{s}$ are used, with the nature of $\phi$ identified in the captions, and $z_{s}$ being simply a generic spatial abscissa. The synthetic signals chosen and the resulting velocity $u=e \times x$ are as follows:

$$
\begin{aligned}
& x\left(z_{s}\right)=\sin \left(2 \pi f_{H F} z_{s}\right), \\
& e\left(z_{s}\right)=1+2 \times \frac{u_{L S}}{\langle U\rangle}, \\
& u\left(z_{S}\right)=\sin \left(2 \pi f_{H F} z_{s}\right) \times\left(1+2 \times \frac{u_{L S}}{\langle U\rangle}\right),
\end{aligned}
$$

where $f_{H F}$ is the high-frequency signal emulating turbulent SS fluctuations, and $\langle U\rangle$ is the mean velocity at the channel-flow location from which the LS component $u_{L S}$ was taken. The signal $u\left(z_{s}\right)$ is shown by the black line in Figure 4(a). This signal is symmetric, and its PDF is likewise symmetric.

The blue lines in Figure 4(a), one the mirror image of the other, are the envelopes of the SS signal, as determined from the application of the Hilbert transform of Mathis et al. The process starts with the definition of the augmented signal of $u$, involving the Hilbert transform $\breve{u}$ (item 1 in Section I)

$$
\begin{aligned}
z & =u+i \breve{u} \\
& =x e+i \widetilde{x e},
\end{aligned}
$$

the latter being consistent with non-overlapping spectra for $x$, which has a high frequency, and $e$, which has a low frequency and is associated with the LS motions. Subject to non-overlapping 


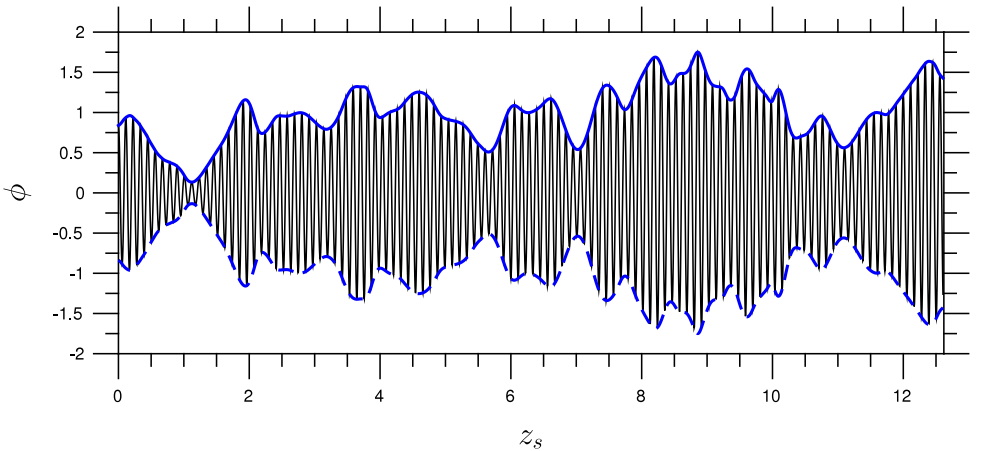

(a)

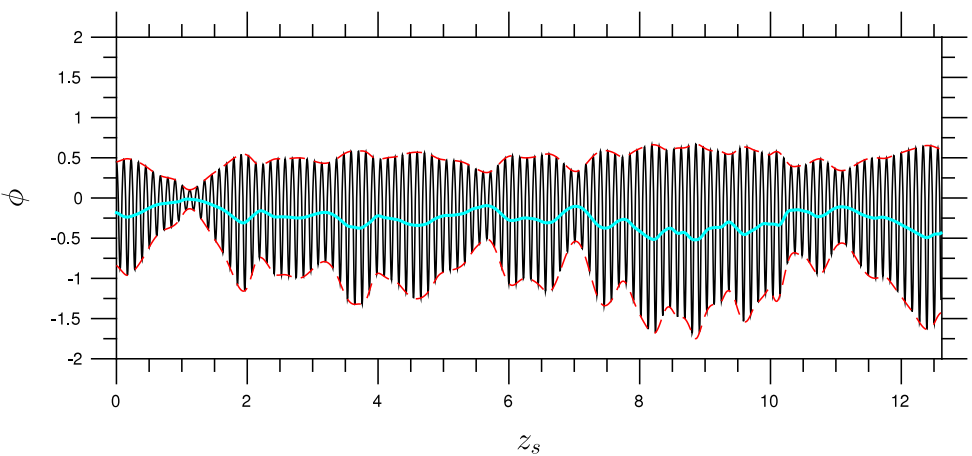

(b)

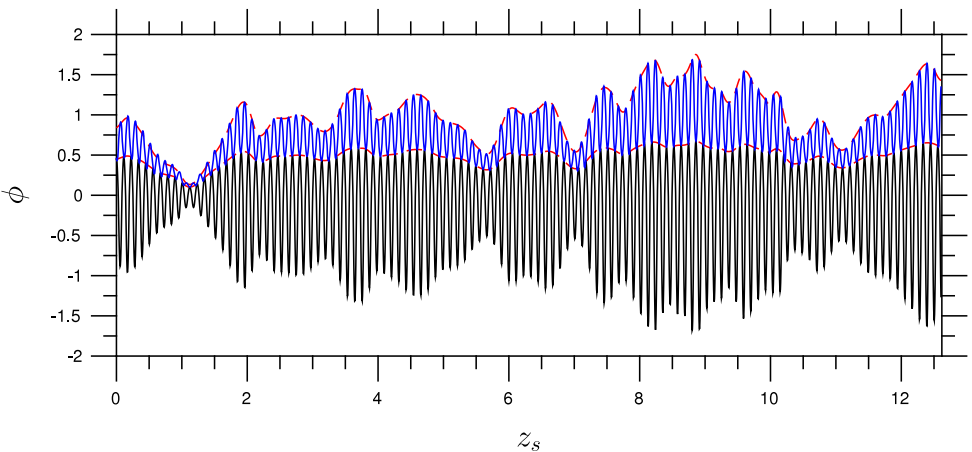

(c)

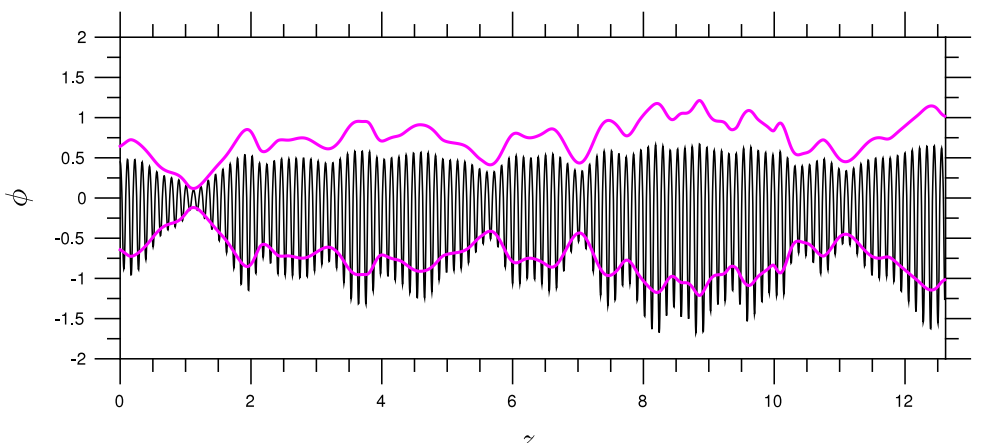

(d)

FIG. 4. Synthetic high-frequency sinusoidal signal modulated by the large-scale signal of the channel flow shown in Figure 3. (a) Symmetric modulation; (b) asymmetric modulation with negative skewness (cyan line) imposed on the positive fluctuations; (c) the modulus of the augmented signal $z$ obtained from the Hilbert transform of the skewed signal (blue line); (d) the envelope of the skewed signal (upper magenta line) following low-pass filtering of the modulus of the augmented signal $|z|$ (lower magenta line is the mirror image of the upper line). 
spectra, Bedrosian's theorem ${ }^{17}$ allows the Hilbert transform of $u$ to be rewritten such as $\breve{x e}=\breve{x} e$. Therefore, the augmented signal may be expressed by

$$
z=e \times(x+i \breve{x}) .
$$

The envelope of the SS fluctuations is determined from the modulus of $z$,

$$
\begin{aligned}
|z| & =\sqrt{u^{2}+\breve{u}^{2}} \\
& =\sqrt{(x e)^{2}+(\breve{x e})^{2}} \\
& =e \sqrt{x^{2}+\breve{x}^{2}} .
\end{aligned}
$$

As the signal $x$ is purely sinusoidal, it follows that

$$
\begin{aligned}
|z| & =e \sqrt{\sin ^{2}\left(2 \pi f_{H F} z_{S}\right)+\cos ^{2}\left(2 \pi f_{H F} z_{S}\right)} \\
& =e .
\end{aligned}
$$

Hence, in this particular case, the low-pass-filtering step (item 2 in Section I) is not required, and $|z|=|z|_{L F}=e$, where the subscript $L F$ denotes low-pass filtering.

In order to synthetically mimic the effects of skewness, associated with "splatting," the negative fluctuations of $x$, which are modulated by $e$, are left unchanged, while the positive fluctuations are modulated by $s \times e$, with $s$ prescribed (note that $s$ is not the skewness itself, but a representative thereof). To enhance realism, within the synthetic framework, $s$ is chosen by reference to observations of how the skewness depends on the LS motions, i.e., $s$ is allowed to vary with the LS motions.

Figure 2(a) shows that the sign of the conditional skewness changes across the buffer layer, but that the location of this switch depends sensitively on the LS motion, especially within the buffer layer. At $y^{+} \approx 12.5$ - the location to which Figures 1 and 2 relate-strongly negative skewness is associated with strongly positive LS motions, while skewness is low for strongly negative LS motions. This is the background against which $s$ was sensitized as follows to the LS signal, whilst remaining (intentionally) unconditionally negative:

$$
s=\left(2+1.6 \times \frac{u_{L S}}{\langle U\rangle}\right)^{-1} .
$$

This signal is shown in Figure 4(b) by the cyan line, and this is also identical to the result of low-pass filtering the signal $u\left(z_{s}\right)$. With $s$ so defined, the resulting signal $u$ arises as follows:

$$
\begin{aligned}
u & =e\left(s \frac{(\operatorname{sgn}(x)+1)}{2} x-\frac{(\operatorname{sgn}(x)-1)}{2} x\right) \\
& \equiv e\left(s f_{1}+f_{2}\right),
\end{aligned}
$$

in which $f_{1}$ and $f_{2}$ are associated with positive and negative SS fluctuations, respectively. The resulting skewed signal is shown in Figure 4(b) by the black line, the envelopes of which arise from those in Figure 4(a) upon adding $s$ to the upper envelope. As both $s$ and $e$ have a low frequency, while $f_{1}$ and $f_{2}$ are at high frequency, Bedrosian's theorem allows the Hilbert transform of $u$ to be determined from

$$
\breve{u}=e\left(s \breve{f}_{1}+\breve{f}_{2}\right)
$$

The modulus $|z|$ then arises from

$$
\begin{aligned}
|z| & =\sqrt{u^{2}+\breve{u}^{2}} \\
& =\sqrt{\left(e s f_{1}+e f_{2}\right)^{2}+\left(e s \breve{f}_{1}+e \breve{f}_{2}\right)^{2}} \\
& =\sqrt{e^{2} s^{2}\left(f_{1}^{2}+\breve{f}_{1}^{2}\right)+e^{2}\left(f_{2}^{2}+\breve{f}_{2}^{2}\right)+2 e^{2} s\left(f_{1} f_{2}+\breve{f}_{1} \breve{f}_{2}\right)} \\
& =\sqrt{e^{2}\left[\left(s f_{1}+f_{2}\right)^{2}+\left(s \breve{f}_{1}+\breve{f}_{2}\right)^{2}\right]} .
\end{aligned}
$$


The modulus $|z|$ derived from the skewed signal $u$ and its Hilbert transform are represented by the blue line in Figure 4(c). This has the appearance of a modulated signal having a frequency identical to the original signal $u$. Following the proposal of Mathis et al., $|z|$ is now low-pass filtered, based on the assumption that the high-frequency component of $|z|$ is noise, and the envelope arises as

$$
|z|_{L F}=\frac{1}{2} e(s+1)
$$

which is represented by the magenta line in Figure 4(d). This is now regarded as a unique representative of the modulation, both for positive and negative SS fluctuations. However, this result clearly provides only an approximation of the modulation.

In order to recover a representation that accounts for the skewness, it is proposed here to perform a second Hilbert transform (identified by $w$ ) on a "residual" signal $r\left(z_{s}\right)$, defined as the difference between the blue line in Figure 4(c), $|z|$, and its low-pass-filtered magenta envelope, $|z|_{L F}$, in Figure 4(d), i.e.,

$$
\begin{aligned}
r & =|z|-|z|_{L F}, \\
w & =r+i \breve{r}, \\
|w| & =\sqrt{r^{2}+\breve{r}^{2}}, \\
|w|_{L F} & =\frac{1}{2} e(1-s),
\end{aligned}
$$

in which $w$ is the augmented signal involving the Hilbert transform of the residual, and $|w|_{L F}=|w|$, because the residual is here symmetric and of a single frequency. The envelope of the symmetric signal, shown in Figure 5(a) by the green line, is now combined with the magenta line in Figure 4(d) as explained below.

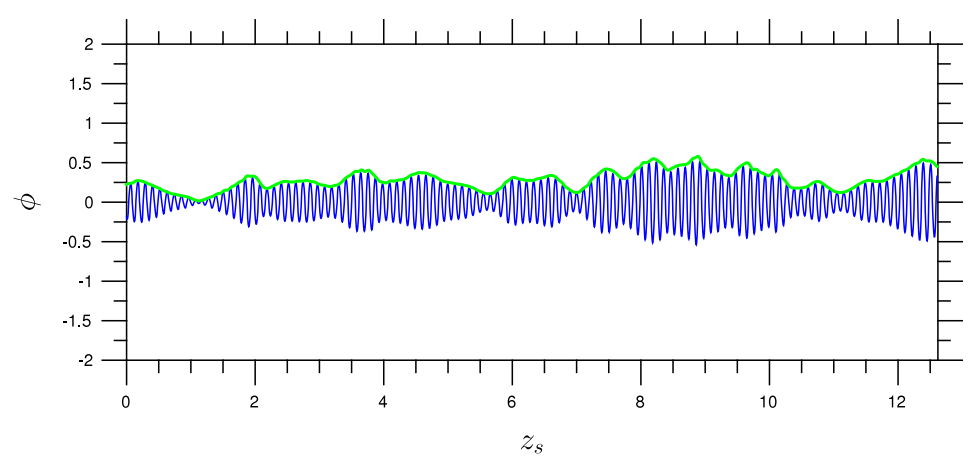

(a)

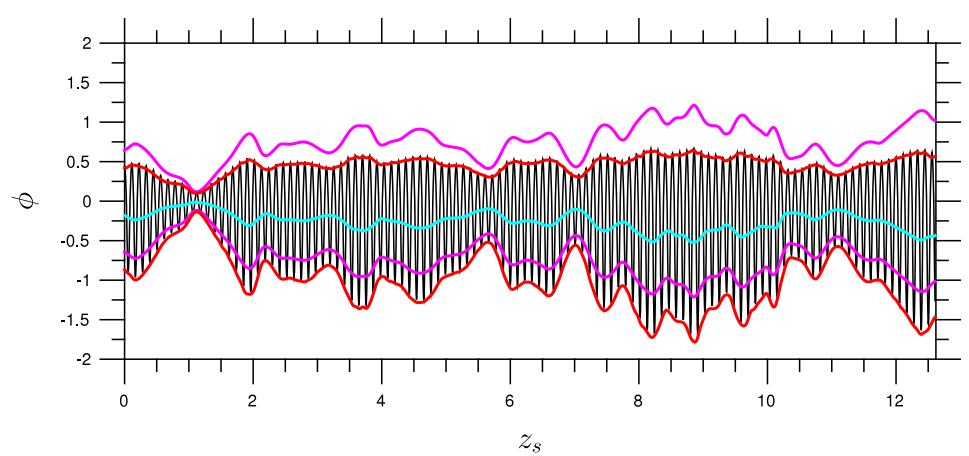

(b)

FIG. 5. Construction of separate upper and lower envelopes of negatively skewed synthetic signal by double Hilbert transform. (a) Residual signal $r$ (blue line) derived from blue signal in Figure 4(c), relative to its low-pass-filtered distribution (magenta line in Figure 4(d)), and the modulus of its augmented signal $|w|$ (green line); (b) the two envelopes of the skewed signal (red lines). 
It is recalled that the cyan line in Figure 4(b) is the low-pass-filtered skewed signal $u\left(z_{s}\right)$. The sign of this distribution provides information on the variation of the "local" skewness, here negative, but in general positive or negative. For regions of negative "local" skewness (dictated by $u_{L F}$ ), the upper envelope of the high-frequency signal is obtained by subtracting the residual envelope $|w|_{L F}$ (shown in Figure 5(a) by the green line) from the lower magenta line $|z|_{L F}$ in Figure 4(d), while the lower envelope arises from adding the envelope of the residual to the upper magenta line. If the skewness were positive, the process would be reversed, and it may thus be expressed collectively by

$$
\begin{aligned}
& E_{\text {upper }}=|z|_{L F}+\operatorname{sign}\left(u_{L F}\right) \times|w|_{L F}, \\
& E_{\text {lower }}=-|z|_{L F}+\operatorname{sign}\left(u_{L F}\right) \times|w|_{L F} .
\end{aligned}
$$

The envelopes resulting from this process are shown in Figure 5(b) by the red lines. The point to highlight is that there are now two different envelopes, one associated with positive high-frequency fluctuations and the other with negative ones. There are, therefore, two associated correlation coefficients between the LS motions and the modulated SS motions.

A question to address next is how the above process should be modified to cater for a skewness variation that changes in sign. This condition poses a significant challenge, as changes in sign in Equations (17) and (18) tend to cause discontinuities in the envelopes. To mimic such a condition, the skewness parameter $s$, earlier defined by Equation (8), is modified as follows:

$$
s=\left(1+1.6 \times \frac{u_{L S}}{\langle U\rangle}\right)^{-1} .
$$

This distribution is shown by the cyan line in Figure 6(a), together with the envelopes (corresponding to those in Figure 4(b)). As before, the cyan line is the low-pass-filtered form of $u\left(z_{s}\right)$. The result of applying the method of Mathis et al. is given in Figures 6(b) and 6(c), the former showing the envelope $|z|$ (blue signal), and the latter its low-pass-filtered form $|z|_{L F}$, constituting the envelope of Mathis et al. (magenta lines).

If, alternatively, the present method is applied, by way of Equations (17) and (18), the result is that shown in Figure 7(a) by the red lines. Clearly, the fidelity of the envelopes is inferior to that in Figure 5(b). A more serious problem is the appearance of discontinuities that arise at sign-switching locations.

In an effort to improve the representation of the envelopes, an alternative method is proposed next. This rests on a separate treatment of the positive and negative parts of the field $u\left(z_{s}\right)$, with the former used to determine the upper envelope and the latter used for the lower envelope. The two partial signals, bounded by zero on one side, are denoted $u_{p}$ and $u_{m}$, respectively. For either signals, Equations (17) and (18) provide two envelopes, one being zero and the other being the envelope at the other extreme of the fluctuations (positive or negative).

Reference to Equations (12) and (16) shows that $|z|_{L F}$ and $|w|_{L F}$ are given by $\frac{1}{2} e(s+1)$ and $\frac{1}{2} e(1-s)$, respectively. Therefore, the imposition of zero value on one side of the partial signal $u_{p}$ or $u_{m}$ is equivalent to setting $s=0$. It follows that $|w|_{L F}=|z|_{L F}$, and the envelope of the residual is no longer required as a separate entity. In specific terms, the upper and lower envelopes of the positive partial signal $u_{p}$ arise from the following derivation, in which $\left|z_{p}\right|_{L F}$ is equivalent to $|z|_{L F}$ and $\left|w_{p}\right|_{L F}$ is equivalent to $|w|_{L F}$ in Equations (17) and (18) (in the Appendix, the process is conveyed by the diagram in Figure 16):

$$
\begin{aligned}
z_{p} & =u_{p}+i \breve{u}_{p}, \\
\left|z_{p}\right| & =\sqrt{u_{p}^{2}+\breve{u}_{p}^{2}}, \\
r_{p} & =z_{p}-\left|z_{p}\right|_{L F}, \\
w_{p} & =r_{p}+i \breve{r}_{p}, \\
\left|w_{p}\right| & =\sqrt{r_{p}^{2}+\breve{r}_{p}^{2}},
\end{aligned}
$$




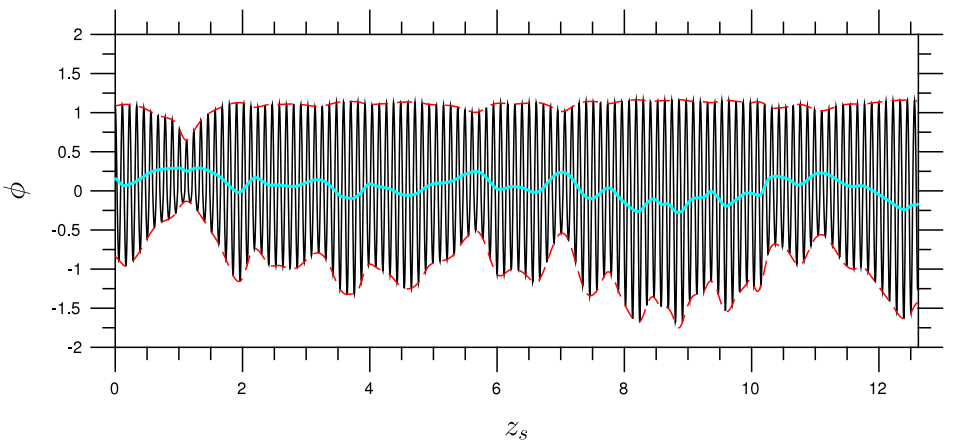

(a)

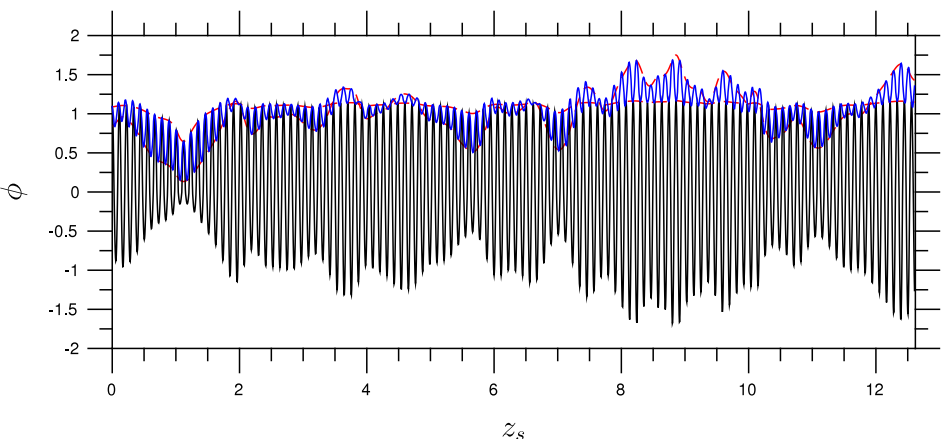

(b)

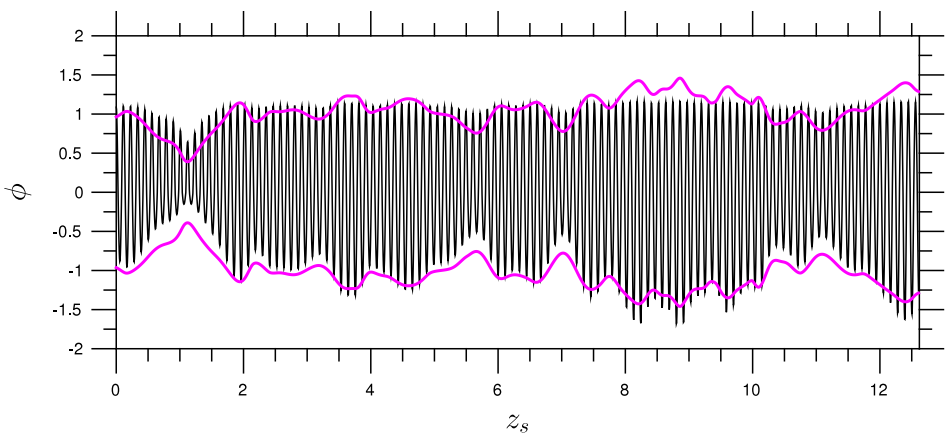

(c)

FIG. 6. Construction of envelopes of synthetic signal skewed by a distribution with negative and positive portions, using the method of Mathis et al. (a) Asymmetric modulation with skewness (cyan line) imposed on the positive fluctuations; (b) modulus of augmented signal $|z|$ (blue line) derived from Hilbert transform; (c) envelopes derived from low-pass filtering of $|z|_{L F}$ (magenta lines).

$$
\begin{aligned}
E_{\text {upper }}\left(u_{p}\right) & =\left|z_{p}\right|_{L F}+\left|w_{p}\right|_{L F} \\
& =\frac{1}{2} e_{u}+\frac{1}{2} e_{u} \\
& =2 \times\left|z_{p}\right|_{L F}, \\
E_{\text {lower }}\left(u_{p}\right) & =-\left|z_{p}\right|_{L F}+\left|w_{p}\right|_{L F} \\
& =-\frac{1}{2} e_{u}+\frac{1}{2} e_{u} \\
& =0 .
\end{aligned}
$$

An entirely equivalent process applies to the derivation of the envelopes of the negative partial signal $E_{\text {upper }}\left(u_{m}\right)$, the end result being 


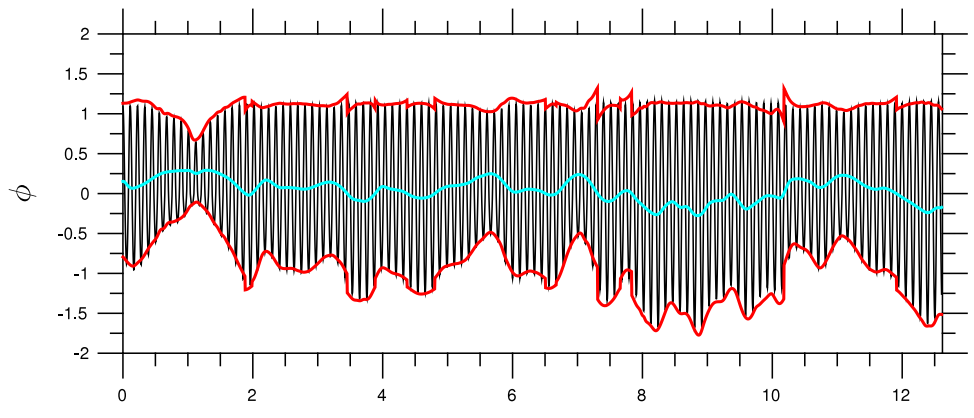

$z_{s}$

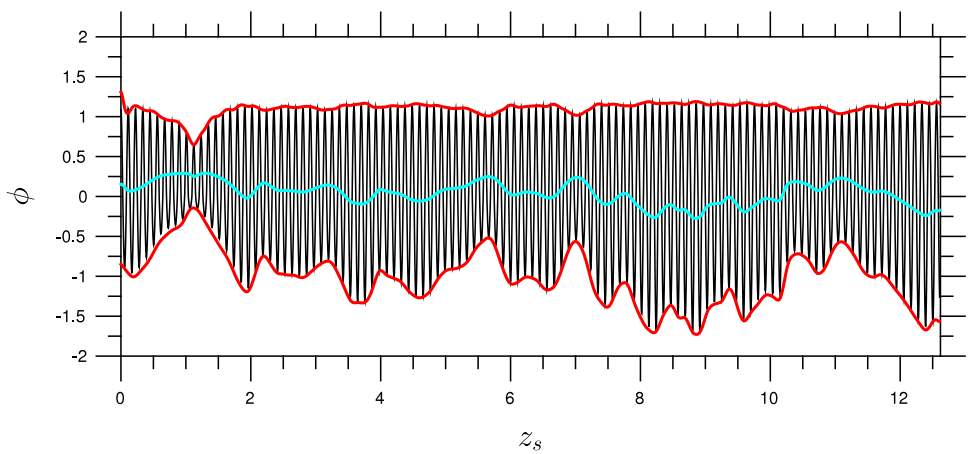

(b)

FIG. 7. Envelopes arising from two versions of the present method (a) derived from Equations (17) and (18) and (b) derived from Equations (24) and (25) (red lines).

$$
\begin{aligned}
& E_{\text {upper }}(u)=E_{\text {upper }}\left(u_{p}\right)=2 \times\left|z_{p}\right|_{L F}, \\
& E_{\text {lower }}(u)=-E_{\text {upper }}\left(u_{m}\right)=-2 \times\left|z_{m}\right|_{L F} .
\end{aligned}
$$

These new envelopes are shown in Figure 7(b) (red lines) and are, as expected, close to the envelopes shown in Figure 7(a), but without the discontinuities in the latter.

\section{THE LINK BETWEEN THE CORRELATION COEFFICIENT AND THE SKEWNESS}

Mathis et al. ${ }^{4,13,18}$ have shown that the correlation coefficient between the envelope of the SS field and the LS motions decreases from a high value of around 0.5-0.8 at $y^{+}<6$ up to around zero at $y^{+} \approx 20$, followed by a slight increase until the end of the buffer layer and a change to a negative value within the log-law region. Reference to Figure 2(a) reveals qualitative similarities between the variation in correlation coefficient and that of the skewness, and this might be taken to suggest a causal relationship between the two. Metzger and Klewicki ${ }^{19}$ show, however, that the skewness level and the details of its variation depend on the Reynolds number, and this implies that the variation in the skewness is sensitive to the LS motions.

As noted in the Introduction, the above similarities have led Schlatter and Örlü and Bernardini and Pirozzoli ${ }^{9,10}$ to examine the dependence of the correlation coefficient on the skewness. The present section discusses this link against the background, provided in Section III, on the dependence of the envelope of the SS field (determined with the method of Mathis et al.) on the skewness, and on the fact that the local skewness depends on the LS motions.

The correlation coefficient is given by

$$
R_{u_{L S},|z|_{L F}}=\frac{\operatorname{cov}\left(u_{L S},|z|_{L F}\right)}{\sqrt{\sigma_{u_{L S}} \sigma_{|z|_{L S}}}} .
$$


It was shown in Section III B that the envelope of a skewed signal, determined with the method of Mathis et al., is $|z|_{L F}=\frac{1}{2} e(s+1)$. For the following argument, it is sufficient to assume here that the correlation between the envelope and the LS motion is very high, so that $e \approx u_{L S}$. It then follows that

$$
\begin{aligned}
R_{u_{L S},|z|_{L F}} & =\frac{\operatorname{cov}\left(e, \frac{1}{2}(e(s+1))\right)}{\sqrt{\sigma_{e} \sigma_{\frac{1}{2}(e(s+1))}}} \\
& =\frac{\operatorname{cov}(e, e)}{\sqrt{\sigma_{e} \sigma_{e(s+1)}}+\frac{\operatorname{cov}(e, e s)}{\sqrt{\sigma_{e} \sigma_{e(s+1)}}} .} .
\end{aligned}
$$

The above equation makes clear that the correlation coefficient depends on local skewness variations, in the sense of Equation (8) or (19). If the skewness is constant-say, $s=A$, whatever the constant $A$-the correlation coefficient is 1 . Only if the skewness depends on the LS motions-as it does in reality-does the correlation coefficient decrease. This is not in agreement with the observations of Schlatter and Örlü, ${ }^{9}$ which associate the increase/decrease of the correlation purely with the increase/decrease in the skewness itself.

In examining a skewed random (synthetic) signal, Schlatter and Örlü show that a high correlation coefficient is associated with high skewness, but this appears to be a result of the fact that the low-pass-filtered form of the signal is driven purely by skewness variations, and therefore does not represent true LS motions. The consequence is that the local skewness of the modulating signal is constant and is thus divorced from what is regarded as being the LS signal.

An alternative view is provided by Bernardini and Pirozzoli ${ }^{10}$ who focus on the two-point covariance $C_{A M}^{2 p}\left(y_{1}, y_{2}\right)=\operatorname{cov}\left(L S\left(y_{1}\right),|z|_{L F}\left(y_{2}\right)\right)$, with $|z|_{L F}$. Using a random signal with skewness similar to that of the true fluctuation field signal, they show that the correlation between $\operatorname{LS}\left(y_{1}\right)$ in the outer region and $|z|_{L F}\left(y_{2}\right)$ in the inner region $\left(y^{+}=15\right)$ is essentially zero, while the actual (true) signal gave a high correlation value (0.25). From this, Bernardini and Pirozzoli concluded that the skewness was not related to the elevated correlation and was not an influential property. However, if the skewness imposed on the random signal was chosen by Bernardini and Pirozzoli-as it appears to have been-to be constant (i.e., independent from the LS motion), then the claim made by them regarding the insensitivity of the two-point covariance to the skewness supports, prima facie, the present argument that only LS-dependent skewness variations lead to a valid description of the sensitivity of the correlation to the skewness.

\section{APPLICATION TO TURBULENT CHANNEL FLOW}

This section discusses the differences between the present approach and that of Mathis et al. when both are applied to true turbulence fields derived from the DNS data of Lozano-Durán and Jiménez. ${ }^{14,15}$

Figure 8 conveys the present envelope-definition process by reference to the actual turbulent signal given in Figure 3(b) - a one-dimensional, spanwise snapshot of the streamwise SS fluctuations at $y^{+}=12.5$. Figure 8 (a) shows the conventional envelope of the SS signal. This envelope arises from the application of the method of Mathis et al., and it corresponds to the envelope shown in Figure 4(d) for the toy signal. The method does not account for the skewness, and the definition of the envelope is thus not sensitive to whether the small-scale fluctuations are positive or negative. While it is not easy to judge, even qualitatively, whether there is a clear relationship between the envelope and the turbulent signal, there are several intervals that can be identified, both in the positive and negative SS regions, in which the envelope does not represent the amplitude variation of SS fluctuations well.

Figure 8(b) shows the envelope of the "residual" signal, corresponding to that in Figure 5(a), and its envelope $\left(|z|-|z|_{L F}\right.$ in Equation (13)). Unlike the synthetic residual, that considered here is not symmetric and not of single frequency. Hence, the modulus of the augmented signal $w$, Equation (16), needs to be low-pass-filtered, following the second Hilbert transform, to obtain the green envelope; in other words, here, $|w|-|w|_{L F} \neq 0$. 


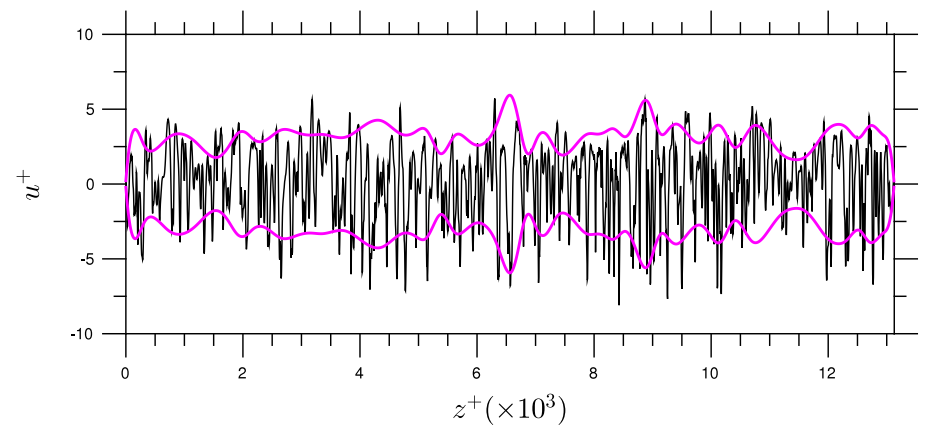

(a)

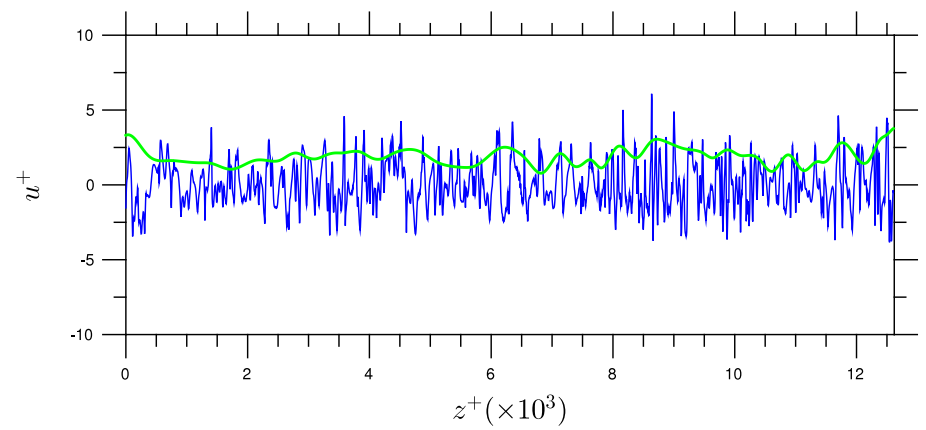

(b)

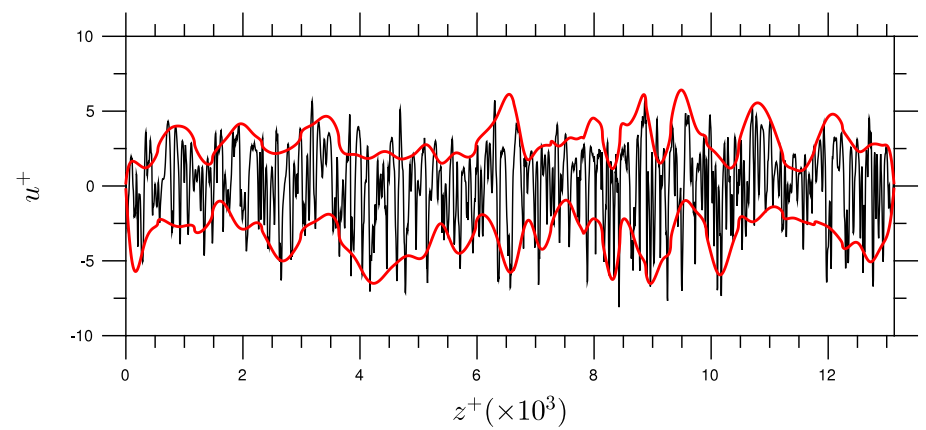

(c)

FIG. 8. Envelopes derived for the snapshot of streamwise SS fluctuations given in Figure 3(b). (a) Mathis et al.'s method, $|w|_{L F}$ (magenta lines); (b) the residual $\left(r=|z|-|z|_{L F}\right)$, (blue line) and its envelope $|w|_{L F}$ (green line); (c) the upper and lower envelopes derived from Equations (24) and (25) (red lines).

Figure 8(c) shows the result of adding/subtracting the envelope of the residual to/from the envelopes shown in Figure 8(a), yielding the two separate red envelopes. A comparison with the envelopes in Figure 8(a) suggests, qualitatively, a significant improvement in the amplitude variations for positive as well as negative SS fluctuations.

Figure 9 shows, for a single snapshot, three views of the streamwise-fluctuations field at $y^{+} \approx 12.5$, over one-half of the $x-z$ simulation domain. Figure 9(a) gives the total fluctuations field, while Figures 9(b) and 9(c) convey the decomposed SS and LS motions, respectively. Close inspection of the SS field, when contrasted with the LS map, reveals that the SS fluctuations are elevated in regions of large positive LS motions and depressed in regions of large negative LS motions. This modulation reveals itself more clearly below, when the envelopes of the SS field and the correlation coefficients between the large-scale motions and the envelopes are examined.

Figure 10(a) shows the envelope determined by applying the method of Mathis et al. to the SS field, and this reveals a clear qualitative correlation between the envelope and the large-scale field in Fig. 9(c). Figure 10(b) gives the envelope of the residual, corresponding to the green line in Figure 8(b). The method of Mathis et al. disregards this field altogether, the implication being that it 


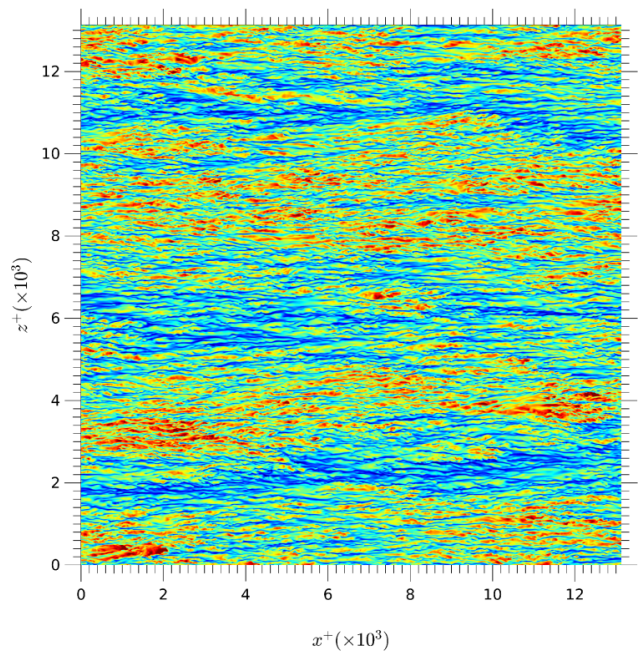

(a)

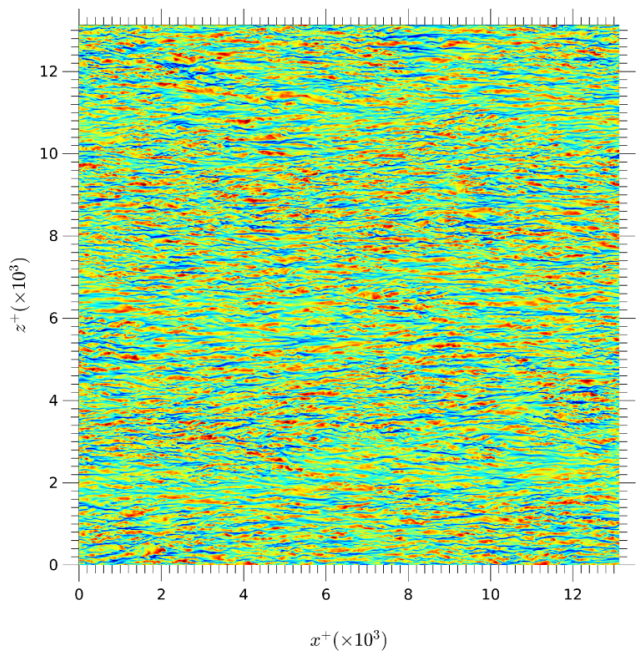

(b)

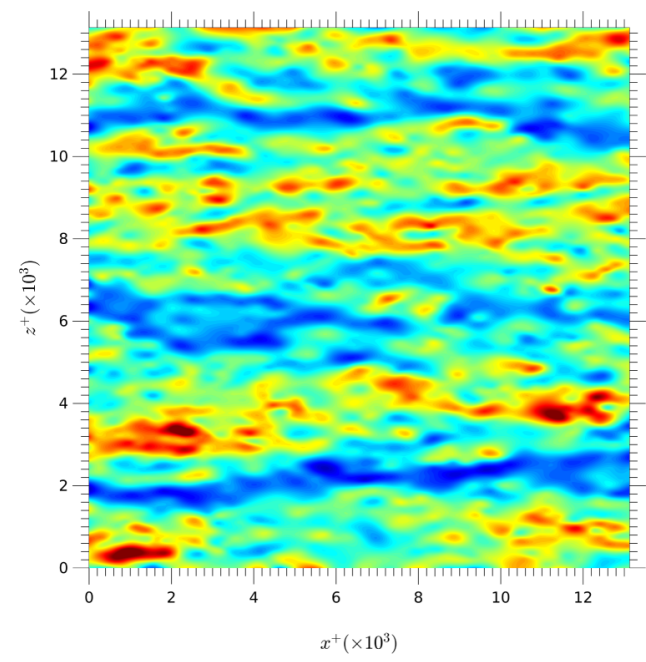

(c)

FIG. 9. Streamwise-fluctuations field in the channel flow at $y^{+} \approx 12.5$. (a) Total fluctuations field, (b) SS fluctuations field, and (c) LS fluctuations field.

represents noise that is uncorrelated to the large-scale motion. This is clearly incorrect, however, as there is a distinct correlation between this envelope and the LS field shown in Fig. 9(c).

The present method, used to determine the lower and upper envelopes of the spanwise SS signal in Figure 8(c), is now applied to two-dimensional snapshots at various $y^{+}$levels, in order to compute the correlation coefficient between the LS motions and the modulated small-scale field. The upper and lower envelopes are calculated, respectively, from Equations (24) and (25). For each $y^{+}$level, the correlation coefficient between the envelopes and the LS motions is determined subject to spatial $x$ - or $z$-wise shifts between the two, the resulting maps being shown in Figures 11(a) and 11(b) for streamwise shifts, and Figures 11(c) and 11(d) for spanwise shifts. These correlations were obtained by processing 13 snapshots, separated by $\Delta t^{+} \approx 230$. The lack of smoothness in the contours occurs mainly around near-zero values of the correlation, and this is an inevitable consequence of imperfect convergence. However, it is important to emphasize that any one of the realisations examined separately shows very similar qualitative features to the averaged correlations given in Figure 11. The correlation between the LS motions and the upper envelope is shown in the left-hand-side maps, while the right-hand-side maps relate to the lower envelope. Although there are clearly some significant quantitative differences between the correlation maps derived for 


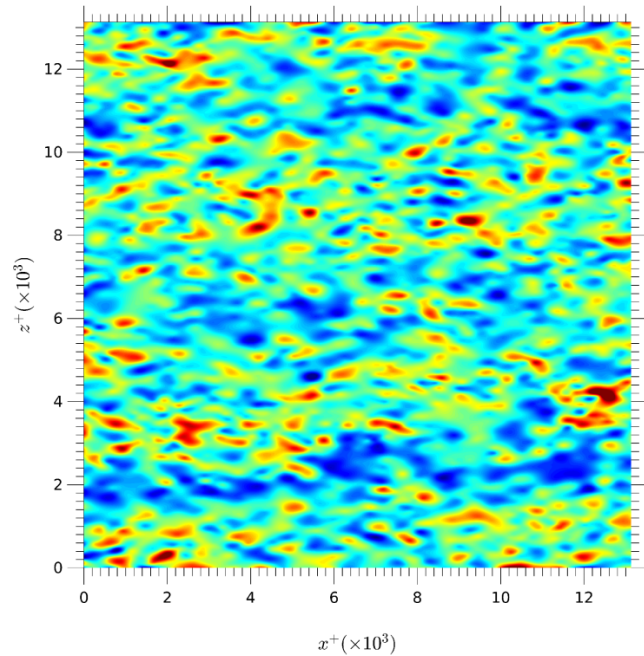

(a)

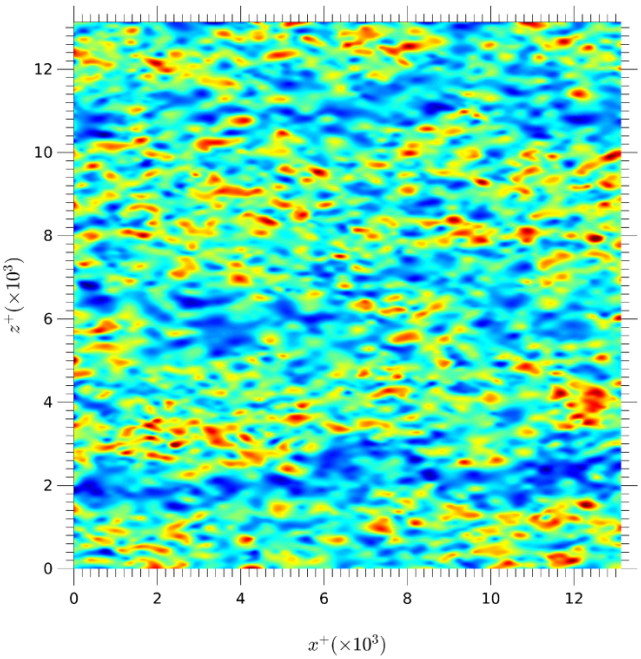

(b)

FIG. 10. Modulation of SS fluctuations field. (a) Envelope obtained with the method of Mathis et al.; (b) envelope $|w|_{L F}$ of the residual $r$ (Equation (13)).

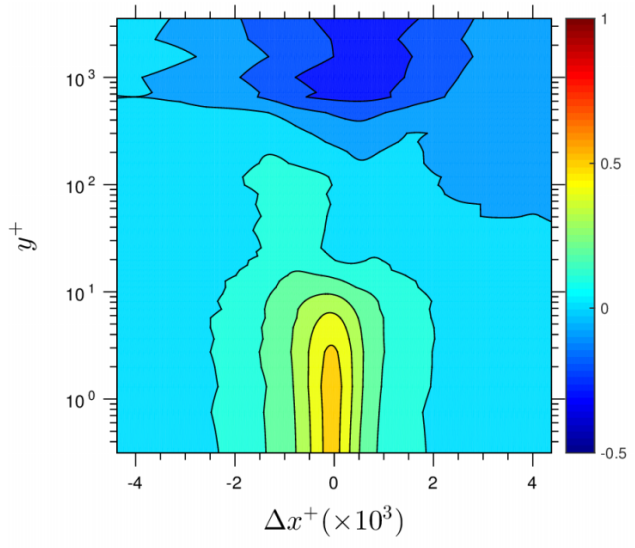

(a)

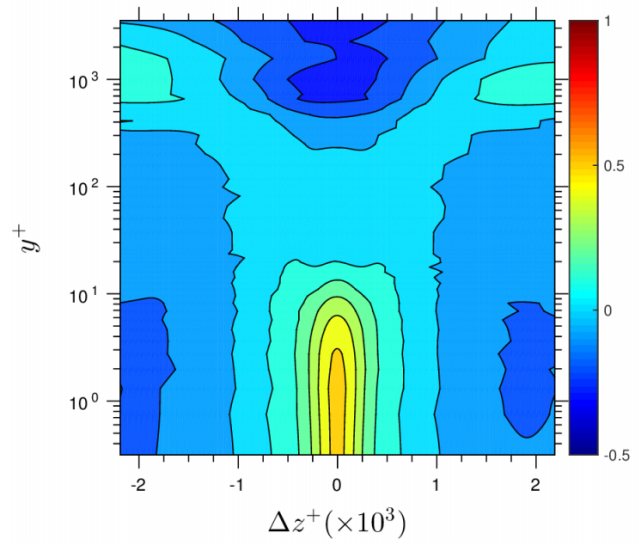

(c)

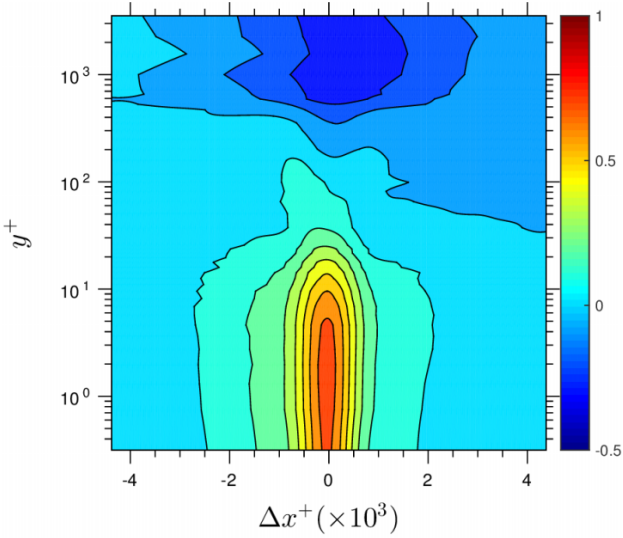

(b)

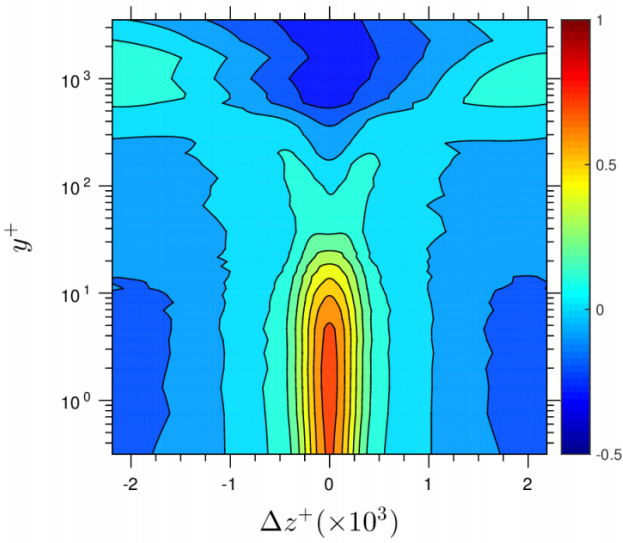

(d)

FIG. 11. Map of correlation coefficient between the LS motions and: (a) and (c) the upper and (b) and (d) lower envelopes. Top line correlation with $\Delta x^{+}$lags and bottom line with $\Delta z^{+}$lags. 
the two envelopes, both demonstrate that the modulation of the SS fluctuations in the near-wall region (below $y^{+}<20$ ) is strongly and positively correlated with the LS motions, the correlation coefficient reaching values of 0.6 very close to the wall, at $y^{+}<8$. In contrast, in the outer region, beyond $y^{+}=250$, the correlation is negative (out of phase) dipping to around -0.4 . Subject to uncertainties arising from differences in the filtering methods, among other processing details, the present results agree broadly with those reported by others, ${ }^{4-9}$ although the latter almost invariably relate to $\Delta x^{+}=\Delta z^{+}=0$ only. However, the magnitude and detailed distribution of the correlation coefficient are clearly sensitive to the nature of the envelope. In particular, Figure 11 makes clear that the upper envelope is more weakly correlated with the LS motion than the lower one, and that the correlation coefficient for the upper envelope decreases faster in the buffer layer than for the lower envelope. These results thus provide added justification for taking the local skewness of the small-scale motions into account, especially as the near-wall skewness, associated with splatting, is highly pertinent to the positive part of the SS field, inducing a diminution in the correlation.

An interesting feature in the maps in Figures 11(a) and 11(b) is the distinct asymmetry, relative to $\Delta x=0$, in the correlation coefficient above $y^{+} \approx 30$. This is especially pronounced in the map pertaining to the lower envelope, which suggests that the switching position varies in the range $y^{+}=40-600$. Chung and McKeon ${ }^{7}$ provide a physical explanation, based on processing of DNS and large-eddy simulation (LES) data, that links the progressive $y^{+}$-wise change in the correlation from positive to negative values to an increasing phase shift between the LS events and SS-signal amplification or attenuation. In particular, a negative correlation is argued to signify, statistically, a phase-shift $\pi$ between the modulation amplitude and the large-scale motion. The change from positive to negative correlation can be expected to be most pronounced along the locus of maximum auto-correlation of the large-scale motions in the $y^{+}-\Delta x^{+}$plane. A map of the this auto-correlation is shown in Figure 12. The maximum of the auto-correlation is observed to occur along a forwardcurving line, and this is the cause of the asymmetry in Figure 11. Between the two peaks in Figure 12 lies a region of depressed auto-correlation, around $y^{+} \approx 50$, and this is also the location at which the correlation coefficient $R$ in Figure 11 is very low, crossing from positive to negative.

Figure 13 shows two maps of the two-point correlation $R_{u_{L S}, E_{S S}}\left(y_{1}^{+}, y_{2}^{+}\right)=\operatorname{cov}\left(u_{L S}\left(y_{1}^{+}\right), E_{S S}\left(y_{2}^{+}\right)\right) /$ $\sqrt{\sigma_{u_{L S}}\left(y_{1}^{+}\right) \sigma_{E_{S S}}\left(y_{2}^{+}\right)}$, pertaining to the correlation of the large-scale motion with the upper and lower modulation envelopes, respectively. The variation along the diagonal is identical to that in Figure 11 at $\Delta x+=\Delta z^{+}=0$. Similar maps have been presented by Bernardini and Pirozzoli ${ }^{10}$ in support of

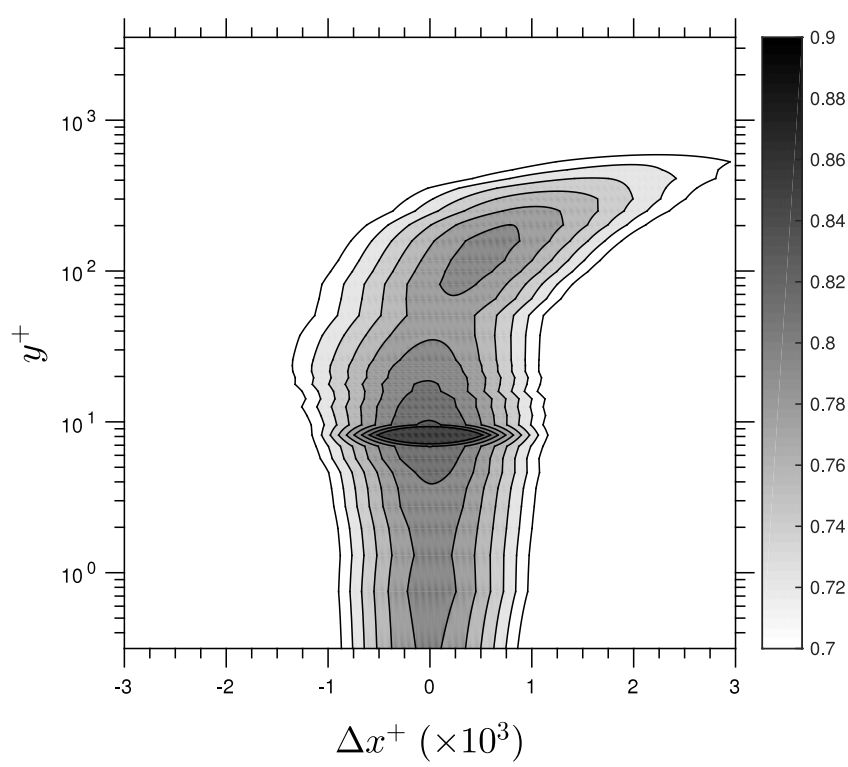

FIG. 12. Two-point correlation map of $u_{L S}$ only, relative to $y^{+}=8$ (the correlation coefficient displayed varies between 0.7 and 0.9). 
the argument that the off-diagonal peak in the correlation coefficient-here along $y_{1}^{+} \approx 150$-is a superior indicator of the effects of the outer large scales on the inner small scales. Figure 13 shows, however, that the correlation coefficient along the diagonal closely approximates the variation along the line $y_{1}^{+} \approx 150$. This is consistent with the fact that the large-scale motions are highly coherent across a substantial portion of the near-wall layer, as shown in Figure 12. The correlation weakens slightly around $y^{+} \approx 50$, creating the impression of a twin-peak topography, but this merely reflects the corresponding twin-peak structure in the autocorrelation map in Figure 12, and it is not related to skewness effects. Finally, the maps show a significant sensitivity to the definition of the modulation envelope, signifying that the correlation level in the off-diagonal region is not divorced from, or is insensitive to, the skewness, as implied by Bernardini and Pirozzoli. ${ }^{10}$

Figure 14 examines fragments of the total skewness and their relationship to the correlation coefficient. As argued by Mathis et al., ${ }^{11}$ the skewness can be written

$$
S_{u} \sigma_{u}^{3}=\overline{u_{L S}^{3}}+3 \overline{u_{L S}^{2} u_{S S}}+3 \overline{u_{L S} u_{S S}^{2}}+\overline{u_{S S}^{3}}
$$

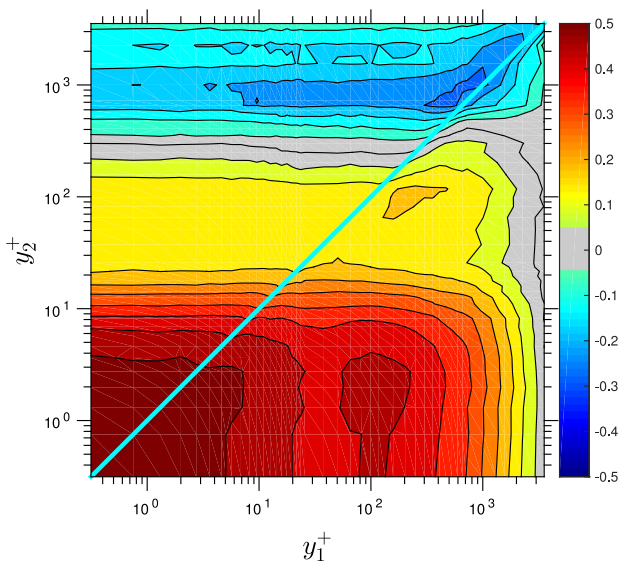

(a)

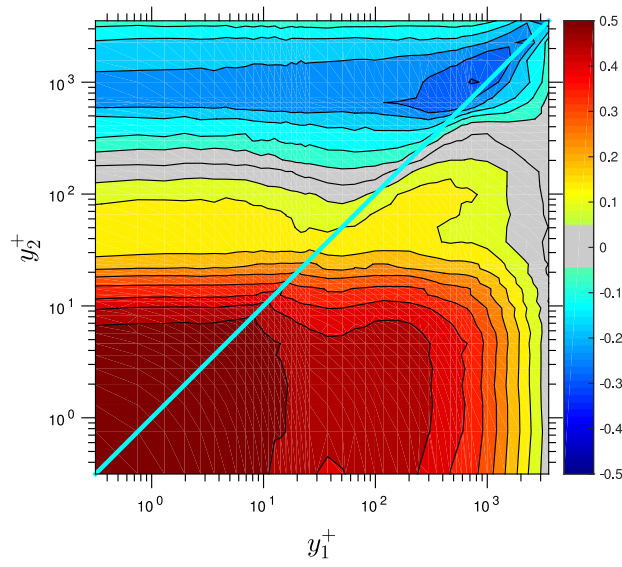

(b)

FIG. 13. Two-point correlation maps $R_{u_{L S}, E_{S S}}\left(y_{1}^{+}, y_{2}^{+}\right)=\operatorname{cov}\left(u_{L S}\left(y_{1}^{+}\right), E_{S S}\left(y_{2}^{+}\right)\right) / \sqrt{\sigma_{u_{L S}}\left(y_{1}^{+}\right) \sigma_{E_{S S}}\left(y_{2}^{+}\right)}$; (a) upper envelope of modulated small-scale signal; (b) lower envelope of modulated small-scale signal.

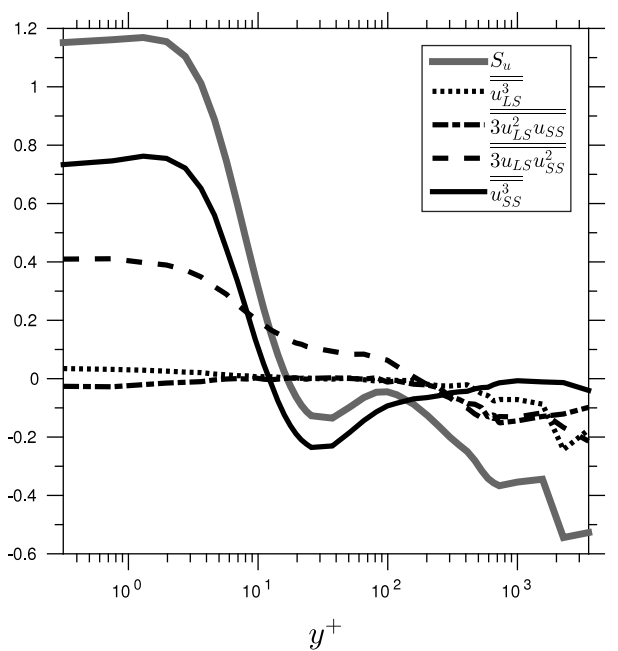

(a)

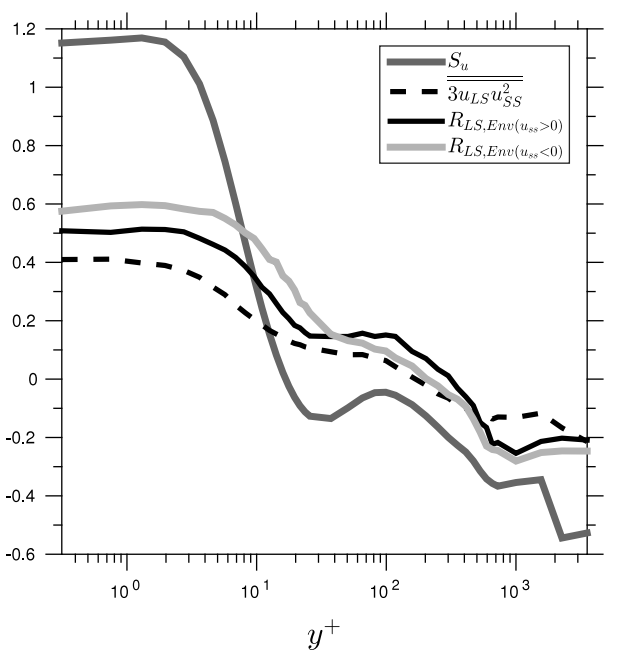

(b)

FIG. 14. Skewness profiles: (a) fragments contributing to the total skewness; (b) relationship between the skewness, its principal mixed-correlation fragment, and the correlation coefficient based on the two envelopes of the small-scale fluctuations. 
These fragments are shown in Figures 14(a) and 14(b) with the notation $\overline{\bar{A}}$ used when $\bar{A}$ is normalized by $\sigma_{u}^{3}$. As noted earlier, Duvvuri and $\mathrm{McKeon}^{12}$ derive a similar relationship from their triadic scale-interaction analysis, with the third term $\left(\overline{u_{L S} u_{S S}^{2}}\right)$ expressed as a scaled correlation coefficient, but they do not provide any information on the relative contributions.

Figure 14(a) gives a breakdown of the contributions to the total skewness, and this shows, in agreement with results by Mathis et al. ${ }^{11}$ that the skewness of the small-scale component dominates over other components. As seen in Figure 14(b), the contribution $\overline{u_{L S} u_{S S}^{2}}$ is well correlated with $R$, an entirely expected behaviour, which justifies statements made by Mathis et al. ${ }^{11}$ and Duvvuri and McKeon ${ }^{12}$ that this fragment may be used as an approximate proxy of $R$. However, this fragment only makes a modest contribution to the total skewness near the wall. By far the largest fragment is the skewness of the small-scale fluctuations. This reflects the fact that positive and negative fluctuations undergo an asymmetric modulation by the low-frequency large-scale motions, with positive fluctuations and associated sweeps being the cause of splatting. The energy redistribution from the streamwise to the spanwise direction accompanying large-scale sweeps cannot be described by the triadic interactions between the small scales. Such interactions can lead to a skewed signal, but this skewness is then induced by an asymmetric modulation at high frequency, and it is thus independent of the large-scale motions. Hence, while the triadic interactions explain how the correlation coefficient is related to the total skewness, this is subject to the implication that the skewness of the small scales is zero.

It is finally noted that Figure 14(b) contains two curves for $R$, one constructed with the upper and the other with the lower envelope of the small-scale signal, and these are identical with the respective variations in Figure 11 at $\Delta x^{+}=0$. While the two curves are qualitatively similar, there are appreciable quantitative differences between them.

\section{CONCLUSIONS}

In order to quantify the relationship - the correlation-between large-scale motions and the modulation of the intensity of the small-scale fluctuations, Mathis et al. ${ }^{4}$ proposed a method for determining the envelope of the small-scale fluctuations. This required the derivation of an augmented signal with the aid of the Hilbert transform of the small-scale fluctuations, followed by low-pass filtering of the modulus of the augmented signal. This method is only effective if the modulation is symmetric and if the small-scale field has zero skewness. Such conditions are rarely, if ever, satisfied. In reality, the skewness is highly negative near the wall and dominated by the small-scale contribution.

The cause of the skewness is an energy redistribution from the streamwise to the spanwise direction, provoked by large-scale sweeps-a phenomenon referred to as a "splatting effect" by Agostini and Leschziner. ${ }^{2}$ Since the positive streamwise fluctuations are preferentially affected by the splatting, the PDF of the small-scale streamwise fluctuations is highly asymmetric and thus skewed. As it is mainly the positive fluctuations that are altered, the skewness becomes increasingly negative as the splatting strengthens. The energy redistribution starts in the viscous sublayer and alters the near-wall state up $y^{+} \approx 30$. Moreover, the splatting strength is large-scale dependent: the stronger the large-scale sweep is, the more intense is the energy redistribution. Hence, the asymmetric modulation associated with the splatting is also large-scale dependent. It follows that low-frequency skewness variations are strongly correlated with the large-scale motions.

The present methodology reflects a recognition of the above interactions and quantifies the effects of skewness on the correlation by defining separate envelopes for the positive and negative small-scale fluctuations. The formalism is an extension and improvement of the method of Mathis et al. In particular, it was shown that the low-pass-filtering of the modulus of the augmented signal, designed to remove noise from the (oscillatory) envelope, amounts to discarding important information that is closely associated with the skewness and the asymmetry of the modulation. The present method includes a step that operates on the augmented signal so as to derive two envelopes, one for positive and the other for negative small-scale fluctuations. Two alternative versions of the new formalism are proposed, and these are summarised diagrammatically in Figures 15 and 16. The 
latter formalism provides a superior definition of the envelopes in the presence of low-frequency skewness variations that involve the "local" skewness changing sign within the domain in which the envelopes are derived.

The application of the formalism to a synthetic signal that is modulated by a skewness-inducing low-frequency signal derived from DNS data demonstrates its validity.

The application of the methodology to channel flow at a friction Reynolds number $R e_{\tau}=4200$ reveals that the envelope of the negative streamwise fluctuations is more highly correlated with the large-scale motions than that of the positive ones. This result is consistent with the observations by Agostini and Leschziner ${ }^{2}$ that splatting affects mainly the positive small-scale fluctuations, which causes perturbations to their amplitude that are less correlated with the large-scale motions.

\section{ACKNOWLEDGMENTS}

The authors are very grateful to Professor J. Jiménez, Dr. A. Lozano-Durán and Dr. J. A. Sillero for sharing their DNS database.

Post-processing work was performed at the Ohio Supercomputer Center.

\section{APPENDIX: DIAGRAMATIC REPRESENTATION OF THE PRESENT ENVELOPE-DEFINITION METHODS}

The process for determining the upper and lower envelopes of high-frequency signal, involving two sequential Hilbert transforms as described to Equations (17) and (18), is summarized in Figure 15.

An alternative version of the present method is conveyed by Figure 16. Here, the calculation of the envelope of the residual is by-passed, and the two envelopes are determined separately, as described by Equations (24) and (25).

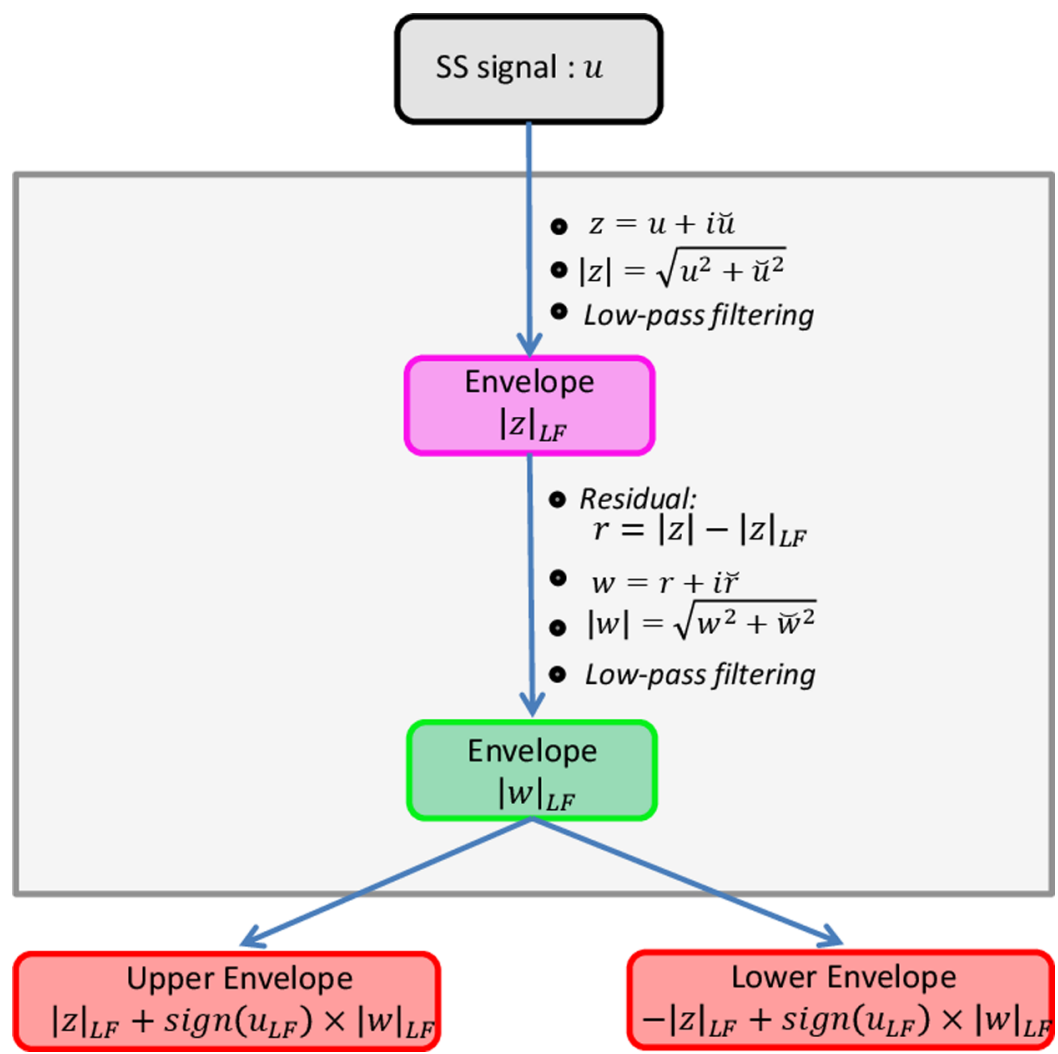

FIG. 15. Diagram summarising default version of present method, Equations (1)-(18). 


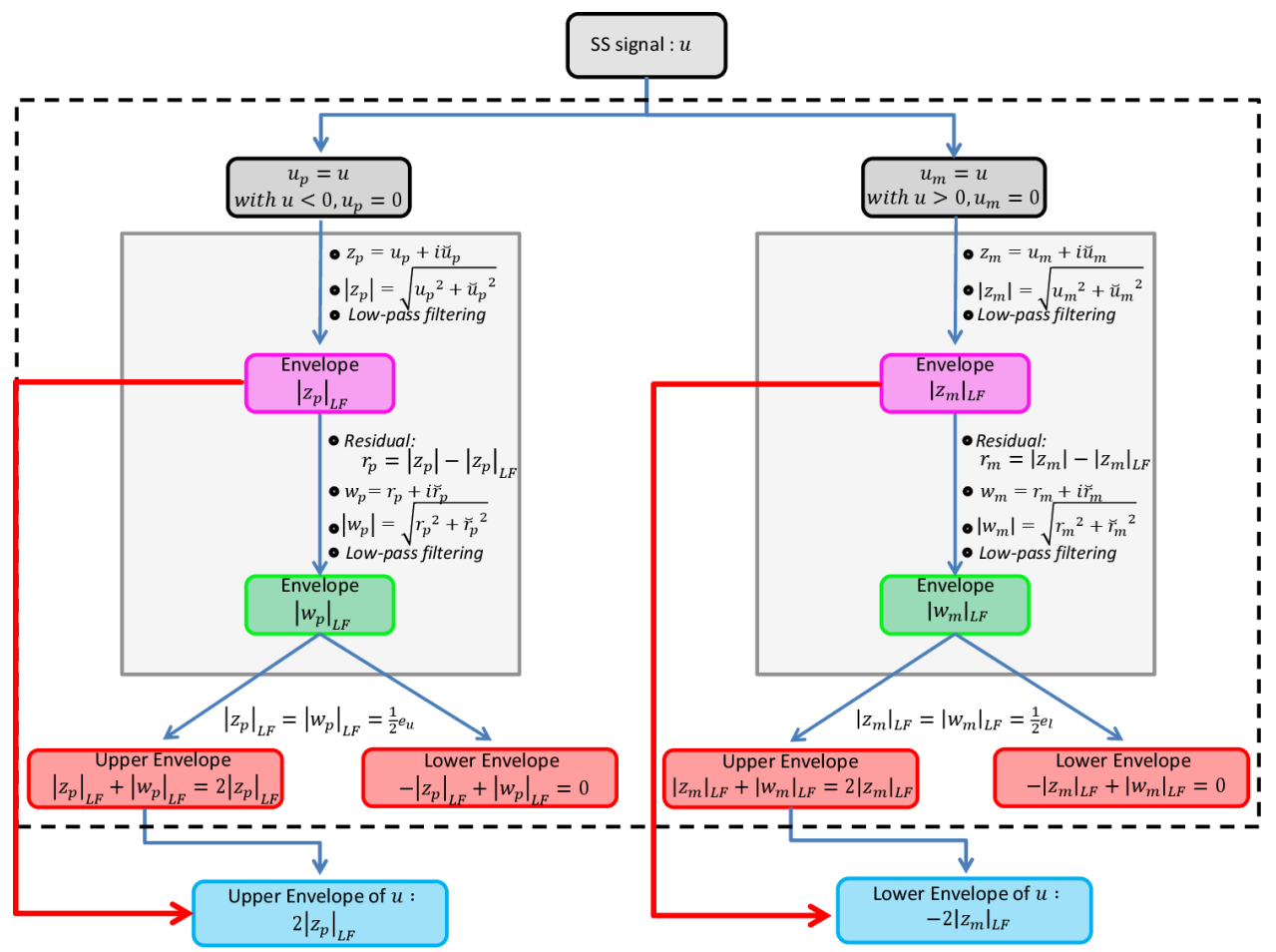

FIG. 16. Diagram summarising alternative version of present method, Equations (20)-(25).

${ }^{1}$ N. E. Huang, Z. Shen, S. R. Long, M. C. Wu, H. H. Shih, Q. Zheng, N.-C. Yen, C. C. Tung, and H. H. Liu, “The empirical mode decomposition and the Hilbert spectrum for nonlinear and non-stationary time series analysis," Proc. R. Soc. A 454, 903-995 (1998).

${ }^{2}$ L. Agostini and M. Leschziner, "On the influence of outer large-scale structures on near-wall turbulence in channel flow," Phys. Fluids 26, 075107 (2014).

${ }^{3}$ P. Bandyopadhyay and A. Hussain, "The coupling between scales in shear flows," Phys. Fluids 27, 2221-2228 (1984).

${ }^{4}$ R. Mathis, N. Hutchins, and I. Marusic, "Large-scale amplitude modulation of the small-scale structures in turbulent boundary layers," J. Fluid Mech. 628, 311-337 (2009).

${ }^{5}$ I. Marusic, R. Mathis, and N. Hutchins, "Predictive model for wall-bounded turbulent flow," Science 329, 193-196 (2010).

${ }^{6}$ K. Talluru, R. Baidya, N. Hutchins, and I. Marusic, "Amplitude modulation of all three velocity components in turbulent boundary layers," J. Fluid Mech. 746, R1 (2014).

${ }^{7}$ D. Chung and B. McKeon, "Large-eddy simulation of large-scale structures in long channel flow," J. Fluid Mech. 661, 341-364 (2010).

${ }^{8}$ I. Jacobi and B. McKeon, "Phase relationships between large and small scales in the turbulent boundary layer," Exp. Fluids 54, 1-13 (2013).

${ }^{9}$ P. Schlatter and R. Örlü, "Quantifying the interaction between large and small scales in wall-bounded turbulent flows: A note of caution," Phys. Fluids 22, 051704 (2010).

${ }^{10}$ M. Bernardini and S. Pirozzoli, "Inner/outer layer interactions in turbulent boundary layers: A refined measure for the large-scale amplitude modulation mechanism,” Phys. Fluids 23, 061701 (2011).

${ }^{11}$ R. Mathis, I. Marusic, N. Hutchins, and K. Sreenivasan, "The relationship between the velocity skewness and the amplitude modulation of the small scale by the large scale in turbulent boundary layers," Phys. Fluids 23, 121702 (2011).

${ }^{12}$ S. Duvvuri and B. J. McKeon, "Triadic scale interactions in a turbulent boundary layer," J. Fluid Mech. 767, R4 (2015).

${ }^{13}$ R. Mathis, J. P. Monty, N. Hutchins, and I. Marusic, "Comparison of large-scale amplitude modulation in turbulent boundary layers, pipes, and channel flows," Phys. Fluids 21, 111703 (2009).

${ }^{14}$ A. Lozano-Durán and J. Jiménez, "Effect of the computational domain on direct simulations of turbulent channels up to $\operatorname{Re} \tau=4200$," Phys. Fluids 26, 011702 (2014).

15 A. Lozano-Durán and J. Jiménez, "Time-resolved evolution of coherent structures in turbulent channels: Characterization of eddies and cascades," J. Fluid Mech. 759, 432-471 (2014).

${ }^{16}$ L. Agostini, E. Touber, and M. Leschziner, "The turbulence vorticity as a window to the physics of friction-drag reduction by oscillatory wall motion,” Int. J. Heat Fluid Flow 51, 3-15 (2015).

${ }^{17}$ P. J. Schreier and L. L. Scharf, Statistical Signal Processing of Complex-Valued Data: The Theory of Improper and Noncircular Signals (Cambridge University Press, 2010).

${ }^{18}$ R. Mathis, N. Hutchins, and I. Marusic, "A predictive inner-outer model for streamwise turbulence statistics in wall-bounded flows," J. Fluid Mech. 681, 537-566 (2011).

${ }^{19}$ M. Metzger and J. Klewicki, "A comparative study of near-wall turbulence in high and low Reynolds number boundary layers," Phys. Fluids 13, 692-701 (2001). 